\title{
Graph-based Criteria for Spectrum-Aware Clustering in Cognitive Radio Networks
}

\author{
Milan Bradonjića , Loukas Lazos, ${ }^{\mathrm{b}, *}$ \\ ${ }^{a}$ Los Alamos National Laboratory, \\ Theoretical Division, and Center for Nonlinear Studies \\ Email: milan@lanl.gov \\ ${ }^{b}$ Department of Electrical and Computer Engineering, \\ University of Arizona, Tucson, Arizona \\ Email:llazos@ece.arizona.edu
}

\begin{abstract}
Cognitive radios (CRs) can exploit vacancies in licensed frequency bands to self-organize in opportunistic spectrum networks. Such networks, henceforth referred to as cognitive radio networks (CRNs), operate over a dynamic bandwidth in both time and space. This inherently leads to the partition of the network into clusters depending on the spatial variation of the primary radio network (PRN) activity. In this article, we analytically evaluate the performance of a new class of clustering criteria designed for CRNs, which explicitly take into account the spatial variations of spectrum opportunities. We jointly represent the network topology and spectrum availability using bipartite graphs. This representation reduces the problem of spectrum-aware cluster formation to a biclique construction problem. We investigate several criteria for constructing clusters for the CRN environment, and characterize their performance under different spectrum sensing and PR activity models. In particular, we evaluate the expected cluster size and number of common idle channels within each cluster, as a function of the spectrum and topology variability. We verify our analytical results via extensive simulations.
\end{abstract}

Keywords: Cognitive radio networks, clustering, graph theory, biclique graphs, bipartite graphs, opportunistic access, open spectrum.

\section{Introduction}

Under a fixed spectrum allocation paradigm, frequency bands are licensed for exclusive use and, in many cases, to specific entities. For example, TV bands are used for the broadcast of TV signals from licensed operators, while public safety radio bands are reserved for radio communications of state, governmental and municipal entities. This paradigm increases the robustness of wireless services by preventing signal interference between different technologies [1,2]. However, measurements of the activity load on the licensed spectrum have shown that a large portion of it is heavily underutilized [3, 4]. To this effect, the Federal Communications Committee (FCC) has recently decided to open up part of the spectrum for unlicensed opportunistic access [5].

${ }^{*}$ Corresponding author. Full Address: Department of Electrical and Computer Engineering, The University of Arizona, 1230 E. Speedway Blvd., Tucson, AZ 85721, USA, Tel: +1-520-626-0736, Fax: +1-520-621-8076. 


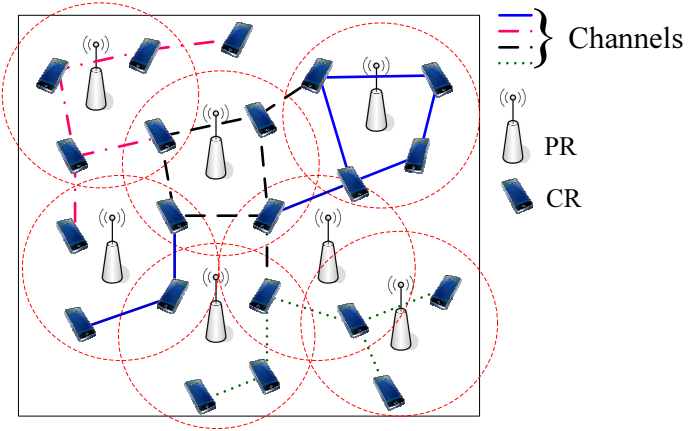

(a)

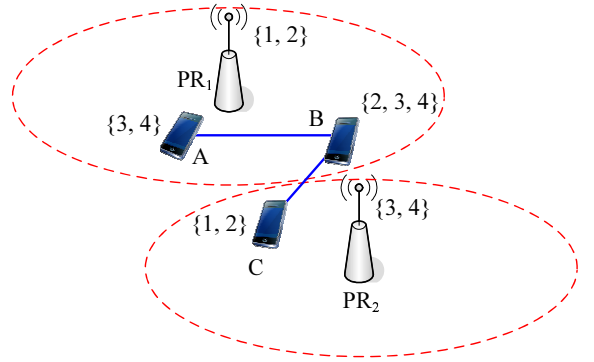

(b)

Figure 1: (a) Co-existence of a CRN with a PRN. The frequency bands exploited by CRs vary in space depending on the ongoing PR activity, (b) the topology of the CRN is dependent on the PR activity. CR nodes $A, C$ cannot communicate because they do not share a common idle channel, despite being within communication range.

Policy regulations dictate that opportunistic users must not interfere with the transmissions of legacy systems [5]. This "no interference" policy leads to a hierarchical network architecture in which licensed users, typically referred to as primary users or primary radios (PRs), have a higher priority in accessing the spectrum compared to unlicensed ones, commonly referred to as secondary users. Cognitive radios (CRs) are one of the most promising technologies for implementing the mandated policy regulations [6]. Using software defined radio technology and an advanced cognition engine, CRs are capable of sensing the idle spectrum either independently, or cooperatively [7-9]. The idle spectrum is then temporarily accessed by the CRs to form a cognitive radio network (CRN).

The unique characteristic of a CRN co-existing with a primary radio network (PRN) is the dynamic nature of the spectrum availability [10]. Consider, for example, the co-existence of a PRN with a CRN, as shown in Figure 1(a). PRN traffic variations lead to a spatial and temporal variation of the CRN topology. Two CR nodes within communication range are not guaranteed to communicate, unless at least one idle band exists at their location. This additional constraint imposes an inherent partition of the CRN into clusters, depending not only on the physical proximity of CRs, but also on the spectrum availability. In this article, we develop and study the performance of clustering criteria that explicitly take into account the spatial variations of the spectrum opportunities.

We note that co-located CRs make correlated sensing observations by sampling the transmission activity of nearby PRs. The set of idle channels ${ }^{1}$ sensed by neighboring CRs varies depending on: (a) the proximity of each CR to active PRs, and (b) the imperfections of the sensing mechanism due to hardware limitations and phenomena of shadowing and fading [10]. For instance, in Figure 1(b), we show three CRs opportunistically accessing a set of four licensed channels. $\mathrm{PR}_{1}$ occupies channels $\{1,2\}$ while $\mathrm{PR}_{2}$ occupies channels $\{3,4\}$. CRs $A$ and $B$ are within the coverage range of $\mathrm{PR}_{1}$ while $C R C$ is within the coverage range of $\mathrm{PR}_{2}$. CRs $A$ and $B$ sense no PR activity on channels $\{3,4\}$ while $C$ senses no PR activity on channels $\{1,2\}$. In addition, $B$ is perceiving channel 2 as idle due to multipath or fading effects. In the CRN of Figure 1(b), $A$, and $C$ cannot directly communicate despite the fact that they are within communication range, because there is no overlap between their respective sets of idle channels.

From the example of Figure 1(b), it becomes evident that the network topology jointly depends on the physical proximity and spectrum availability. Therefore, topology management algorithms such as clustering, must take both these parameters into account. However, we make the observation that clustering criteria

\footnotetext{
${ }^{1}$ In this article, we use the term "channels" to refer to orthogonal frequency bands.
} 
designed for CRNs with dynamic spectrum, may have conflicting goals. On one hand, partitioning the network to a small number of clusters (with larger cluster sizes) reduces the overhead for topology management [11]. On the other hand, grouping a large number of CRs with dissimilar sets of idle channel, reduces the available bandwidth for intra-cluster communication (a smaller number of idle channels is common among all CRs). To capture the aforementioned trade off, we jointly model the physical network topology and spectrum availability at each CR as a bipartite graph. Based on this joint representation, we partition the CRN into clusters by constructing biclique graphs (complete subgraphs of a bipartite graph), which satisfy various design criteria. We initially proposed the idea representing clusters in CRNs as bicliques in [12]. The goal of the work in [12] was to locally allocate common control channels for coordination purposes. The differences between [12] and the present work are summarized in the following contributions.

Contributions. Adopting a graph-based representation of the idle spectrum, we examine three clustering criteria, suitable for CRNs with dynamic spectrum. These criteria are: (a) joint maximization of the sum of common idle channels per cluster with the number of cluster members, (b) joint maximization of the product of common idle channels per cluster times the number of cluster members, and (c) maximization of the number of cluster members under a constraint on the number of common idle channels. We show that our clustering criteria can be combined with clustering algorithms proposed for ad hoc networks, in order to perform spectrum-aware distributed clustering in CRNs. Such clustering, not only allows for enhanced intra-cluster communication due to the availability of multiple common frequency bands, but also inherently implements cooperative spectrum sensing. For each clustering criterion, we analytically evaluate the clustering performance in terms of the feasible clusters, the expected cluster size and the number of common idle channels per cluster. In our derivations, we consider two PR activity models; a semi-Markov ON/OFF model and a Poisson traffic model. However, other traffic models can be incorporated to our analytic results. Furthermore, we consider the clustering process under both perfect and imperfect channel state information. Note that our theoretical evaluation entails the estimation of the feasible bicliques that can be constructed from bipartite graphs with a pre-specified probabilistic structure. Our derivations can be applied to any problem that benefits from a mapping to a biclique representation, and is subject to similar probabilistic models.

Paper organization. The remaining of the paper is organized as follows. In Section 2, we state our system model. In Section 3, we develop a graph model for the joint representation of the physical topology and the idle spectrum availability. Using this model, clusters are mapped to biclique graphs. Spectrum-aware clustering criteria based on the graph representation of clusters are investigated in Section 4. Section 5 discusses algorithms for distributed cluster formation and maintenance. In Section 6 we analytically evaluate the performance of the proposed clustering criteria, and validate our results via extensive simulations. Related work is presented in Section 7. Finally, in Section 8, we present our conclusions.

\section{System Model Assumptions}

For clarity purposes, we first present the notation that will be used in the rest of this article.

\subsection{Notation}

$\mathcal{M}$ : Set of channels licensed to PRNs.

$C_{i}$ : Set of idle channels at CR $i$.

$\mathcal{N}_{i}$ : Set of one-hop neighbors of CR $i$.

$\mathrm{N}$ : Random variable expressing the number of idle channels in the system.

$p$ : Probability that a channel is idle under the semi-Markov activity model.

$p_{m}$ : Probability of mis-detecting the state of an occupied channel. 
$p_{f}:$ Probability of false alarm in sensing an idle channel.

$R: \quad$ PR communication range.

$r:$ CR communication range.

$\lambda$ : Arrival rate for a Poisson-distributed arrival process.

$\mu$ : Departure rate for an exponentially distributed service process.

$\mathcal{G}(\mathcal{A}, \mathcal{B}, \mathcal{E})$ : $\quad$ Bipartite graph $\mathcal{G}$ with sets of vertices $\mathcal{A}, \mathcal{B}$, and set of edges $\mathcal{E}$.

$Q(X, Y)$ : Biclique graph with sets of vertices $X, Y$.

$Z_{\ell}$ : Number of bicliques of "size" $\ell$.

$\ell_{s}:$ Sum of the vertices of a biclique, $\ell_{s}=|X|+|Y|$.

$\ell_{p}:$ Product of vertices of a biclique, $\ell_{p}=|X||Y|$.

$\ell_{c}$ : Cardinality of one side of a biclique, $\ell_{c}=|X|$.

$C(n, k):\left(\begin{array}{l}n \\ k\end{array}\right)$.

\subsection{Primary Radio Network Model}

PRNs are licensed to use a fixed spectrum, which can be divided to a set of orthogonal frequency bands (channels), denoted by $\mathcal{M}=\{1,2, \ldots, m\}$. We assume that all channels are of the same quality in terms of capacity and propagation characteristics ${ }^{2}$. PR users have priority in accessing any channel in $\mathcal{M}$, without experiencing any performance degradation due to transmissions by unlicensed users [5].

\subsubsection{PRN Activity Models}

The proposed clustering criteria and mechanisms do not depend on any particular PRN activity model. However, in order to provide an analytical evaluation of the clustering performance, we consider two PRN activity models that are widely adopted in CRN analysis.

Semi-Markov model. In this model, PRN traffic on a given channel $i \in \mathcal{M}$ is modeled after a semi-Markov ON-OFF process. The state of channel $i$ alternates between busy (ON) and idle (OFF) periods. The length of each period is assumed to be an independent random variable following some distribution. Moreover, the state of a channel $i$ is independent of the state of other channels. This assumption is true when the set of channels $\mathcal{M}$ is licensed to independently operating PRs (e.g., channels operated by different TV stations). Semi-Markov ON-OFF models have been experimentally verified for a range of PRN scenarios $[13,14]$. The semi-Markov renewal process modeling the PRN activity on channel $i$ is shown in Figure 2. Without loss of generality, we assume a geometric distribution on the length of the ON and OFF periods (holding times at each state of the Markov process) with parameters $q_{i}$ and $p_{i}$, respectively. Such assumptions have been extensively adopted in theoretical analyses of the performance of CRNs (e.g., [15-17]). In this case, the stationary probability that channel $i$ is in idle state can be easily computed to be

$$
\pi_{i}^{i d l e}=\frac{q_{i}}{q_{i}+p_{i}} .
$$

Let $\mathrm{N}$ denote the random variable expressing the number of idle channels in the system. Due to the independent channel assumption, the probability mass function (pmf) for $\mathrm{N}$ is given by

$$
\operatorname{Pr}[\mathrm{N}=n]=\sum_{I \subset \mathcal{M}:|I|=n} \prod_{i \in I} \pi_{i}^{i d l e} \prod_{j \notin I}\left(1-\pi_{j}^{i d l e}\right) .
$$

\footnotetext{
${ }^{2}$ CRs may operate over a diverse set of frequency bands with different capacity and propagation characteristics. For simplicity, we assume that there is no preference over the channel of operation.
} 


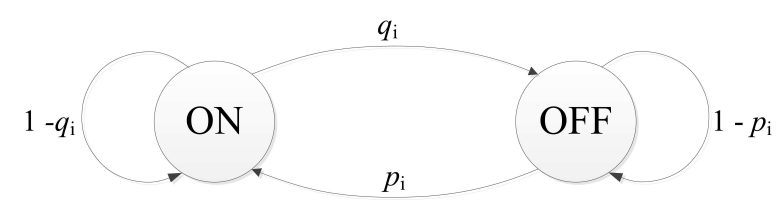

(a)

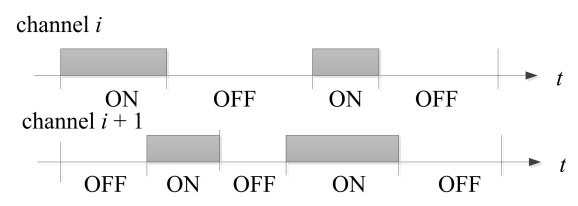

(b)

Figure 2: (a) The semi-Markov PR activity model for channel $i$, (b) PR activity at channels $i$ and $i+1$ modeled as an ON/OFF process.

Assuming that all channels have the same traffic characteristics (i.e., $\pi_{i}^{\text {idle }}=p, \forall i \in \mathcal{M}$ ), (1) is simplified to a binomial distribution

$$
\operatorname{Pr}[\mathrm{N}=k]=\left(\begin{array}{c}
m \\
k
\end{array}\right) p^{k}(1-p)^{m-k}
$$

Poisson model. The second PR activity model aims at capturing the traffic characteristics of PRNs operating under the management of a single entity (for example, a cellular network). In this case, PRN traffic is modeled after a Poisson process with arrival rate $\lambda$ and exponentially distributed service time with mean $\mu[18,19]$. Such assumptions have been verified for networks where the number of users is significantly larger than the number of available channels (as in cellular networks) [18-20]. This model suggests that PRN activity forms an $M / M / c / 0$ queuing system, where $c=m$ denotes the number of channels licensed to the PRN. The stationary probability of $n$ channels being idle ( $m-n$ channels being occupied) is given by the Erlang loss formula [21]:

$$
\operatorname{Pr}[\mathrm{N}=n]=\frac{\frac{\rho^{m-n}}{(m-n) !}}{\sum_{i=0}^{m} \frac{\rho^{i}}{i !}}, n=0,1, \ldots, m,
$$

where $\rho=\frac{\lambda}{\mu}$. In fact, (3) holds true when the channel holding time follows any general distribution $G$ (M/G/c/c queuing model) [21]. The computation of (3) can be simplified using recursion or well known approximations that do not require the calculation of factorials of high order [22].

\subsection{CRN Model}

We assume a decentralized network architecture in which CRs self-organize into a network, without the assistance of a fixed infrastructure. Moreover, no interaction is assumed between the PRNs and the CRN. To prevent interference with the PRs, CRs obtain channel state information in the following two ways: (a) by connecting to a database [5] and, (b) by sensing for idle frequency bands using methods such as energy detectors, cyclostationary feature extraction, and pilot signals [6-9].

Using any of the two methods, each CR $x$ maintains its own set of channels $C_{x}=\left\{i_{1}, i_{2}, \ldots, i_{\left|C_{x}\right|}\right\}$, left idle by the PRN. Here, $i_{j}$ refers to the $j^{\text {th }}$ element of $C_{x}$. When channel state information is obtained via a database, it is assumed to be perfect. On the other hand, when CRs perform their own sensing, channel state information is subject to events of mis-detection and false alarm, due to phenomena of multipath and/or shadowing of PR signals [7-9]. To model the imperfections of the spectrum sensing process, we assume that the state of an occupied channel is falsely estimated with mis-detection probability $p_{m}$. Moreover, the state of an idle channel is falsely estimated (due to, for example, the presence of a large noise component) with false alarm probability $p_{f}$. Given the fast decorrelation of the received signal with space and frequency [23], the events of false alarm and mis-detection are assumed to be independent for each CR and for each channel. Several methods are available for computing typical values of $p_{m}$ and $p_{f}$ [24-26]. 


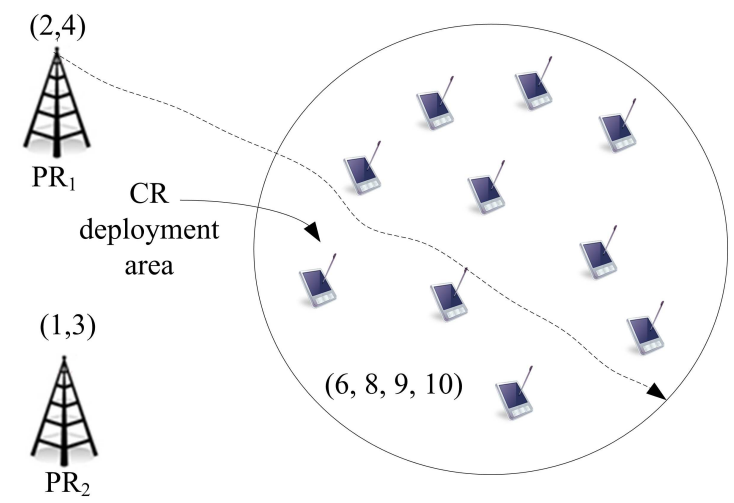

(a) Topology of type-I

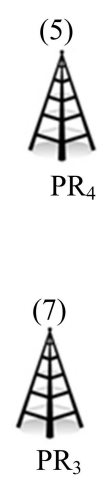

PR

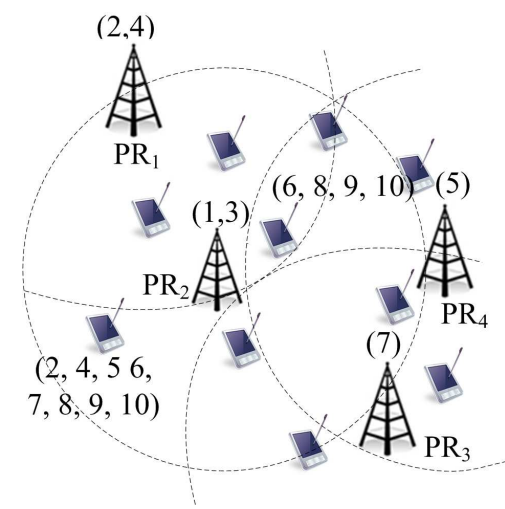

(b) Topology of type-II

Figure 3: Topology architectures modeling the co-existence of the CRN with PRNs. The numbers in parenthesis on top of each PR indicate the channel(s) occupied by that PR. The list of PRs in the CR area indicate the PRs sensed by CRs in that area. For type-I topologies, the set of PRs sensed by each CR is identical. For type-II topologies, neighboring CRs possibly sense different PRs.

\subsection{CRN Topology Models}

Two types of network topologies are considered for the co-existence of the CRN with PRNs. In type-I topologies, the coverage range of PRs denoted by $R$, is significantly larger than the communication range of the CRs, denoted by $r$. Thus, neighboring CRs (within one hop of each other) are within the range of the same set of PRs. This scenario arises when the PRs are powerful base stations such as TV transmitters, with coverage range that spans tens of kilometers [5]. In type-II topologies, the range of the PRs is comparable to that of the CRs. Therefore, neighboring CRs make correlated sensing observations, without necessarily being in the range of the same set of PRs. This scenario arises when the PRs are wireless microphones or cellular towers with limited range [5]. The two types of network topologies are depicted in Figure 3.

The two topological models impose different spatial variation conditions on the set of idle channels at neighboring CRs. For type-I topologies and perfect channel state information, CRs have identical sets of idle channels since they are within the communication range of the same set of PRs. For instance, in Figure 3(a), all CRs within the deployment area sense the activity of $\mathrm{PR}_{1}-\mathrm{PR}_{4}$. Hence, for this scenario, the variation of the idle spectrum is only temporal. For type-II topologies, neighboring CRs are subjected to activity from possibly different sets of PRs. As an example, in the topology of Figure 3(b), CRs $A$ and $B$ have correlated but not identical sets of idle channels. Channel 5 occupied by $\mathrm{PR}_{4}$ is marked occupied by CR $A$, but idle by CR $B$, since $B$ is not within the range of $\mathrm{PR}_{4}$.

To analytically evaluate the proposed clustering criteria, we are interested in modeling the similarity between the sets of idle channels of neighboring CRs. Consider a channel $i$ occupied by a PR $x$. The probability that $i$ is sensed idle by a CR $y$ is dependent upon the distance $d_{x y}$ between $x$ and $y$. For a uniform (random) deployment of PRs and CRs within an area of size $A$, and ignoring any border effects, this probability is equal to [27]:

$$
\operatorname{Pr}\left[d_{x y}>R\right]=1-\frac{\pi R^{2}}{A} .
$$

Equation (4), expresses the probability that CR $y$ is out of the coverage range $R$ of PR $x$. We denote this probability by $p_{w}$. We are also interested in computing the probability that a $\mathrm{CR} z$, neighbor of with CR $y\left(d_{y z} \leq r\right)$, makes a correlated observation on the state of channel $i$. For this, we compute the probability that two CRs sense occupied channel $i$ to be idle, under the constraint that these two CRs are neighbors. We 


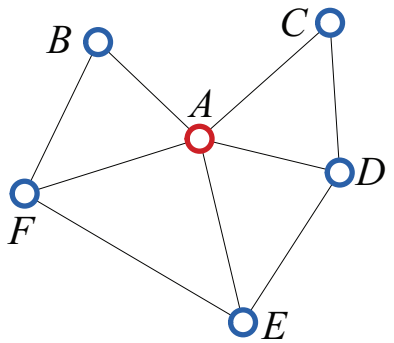

(a)

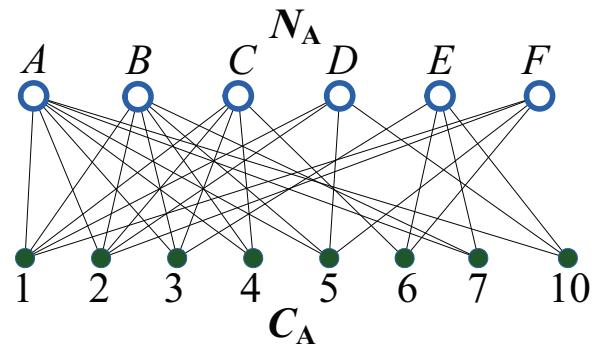

(b)

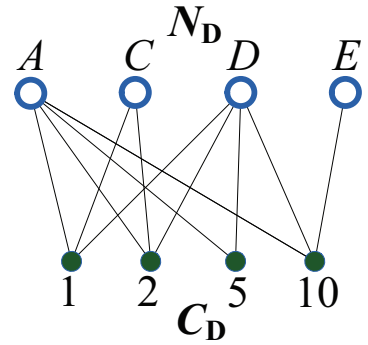

(c)

Figure 4: (a) A CRN of six nodes. The channel availability for each $\mathrm{CR}$ is as follows: $C_{A}=\{1,2,3,4,5,6,7,10\}, C_{B}=$ $\{1,2,3,4,5,7\}, C_{C}=\{1,2,3,4,6,8\}, C_{D}=\{1,2,5,10\}, C_{E}=\{3,6,7,8,10\}$, and $C_{F}=\{1,2,5,6,8\}$, (b) the bipartite graph constructed by node $A$. The two sides of the bipartition are $\mathcal{A}_{A}=A \cup \mathcal{N}_{A}$ and $\mathcal{B}_{A}=C_{A}$, representing the correlation between the channel availability for the neighbors of $A$, (c) the bipartite graph constructed by node $D$.

denote this probability by $p_{c}$.

$$
p_{c}=\operatorname{Pr}\left[d_{x z}>R \mid d_{x y}>R, d_{y z} \leq r\right]=\left(1-\operatorname{Pr}\left[d_{x z} \leq R \mid d_{x y}>R, d_{y z} \leq r\right]\right) .
$$

The value of $p_{c}$ is fixed under a known deployment distribution and fixed values of $R$ and $r$. We derive the exact value of $p_{c}$ for a random CR and PR deployment in Appendix A. The values of $p_{w}$ and $p_{c}$ model the correlation between sensing observations of neighboring CRs. While more elaborate models are possible (different node distributions, sensing models, etc.), this simple model allows us to decouple our analysis from further assumptions regarding the relative locations between the CRs and the PRs. Note that these assumptions are made to facilitate the theoretical analysis of the performance of the clustering algorithms. Our decentralized clustering mechanism does not depend on the validity of such assumptions and can adapt its operation to any set of conditions and topological architectures.

\section{Graph-Based Representation of Idle Spectrum}

In this section, we jointly model the network topology and the idle spectrum availability as a bipartite graph. This model is used as the basis for constructing spectrum-aware clusters. The reasoning behind our model is the co-dependence of the CRN topology on: (a) the communication range of the CR devices, and (b) the overlap between the set of idle channels of neighboring CRs. Both these parameters can be jointly represented by an undirected bipartite graph $\mathcal{G}(\mathcal{A} \cup \mathcal{B}, \mathcal{E})$. A graph $\mathcal{G}(\mathcal{V}, \mathcal{E})$ is called bipartite if the set of vertices $\mathcal{V}$ can be partitioned into two disjoint sets $\mathcal{A}$ and $\mathcal{B}$ with $\mathcal{A} \cup \mathcal{B}=\mathcal{V}$, such that all edges in $\mathcal{E}$ connect vertices from $\mathcal{A}$ to $\mathcal{B}$.

For the purpose of clustering, each CR can construct its own view of the topology/spectrum availability based on the information received from its one-hop neighbors. Let $\mathcal{N}_{i}$ denote the set of one-hop neighbors of a CR node $i$. Assume that CR $i$ collects the sets of idle channels from all CRs in $\mathcal{N}_{i}$. This information can be made available via a cooperative diversity mechanism [7-9]. Then, it can construct a bipartite graph $\mathcal{G}_{i}\left(\mathcal{A}_{i} \cup \mathcal{B}_{i}, \mathcal{E}_{i}\right)$, which jointly represents the similarity between the sets of idle channels of its neighbors and his own set. In graph $\mathcal{G}_{i}, \mathcal{A}_{i}=i \cup \mathcal{N}_{i}$ and $\mathcal{B}_{i}=\mathcal{C}_{i}$. An edge $(x, y)$ exists between a vertex $x \in \mathcal{A}_{i}$ and a vertex $y \in \mathcal{B}_{i}$ if and only if $y \in C_{x}$, i.e., y is common to both $C_{i}$ and $C_{x}$.

As an example, in Figure 4(a), we show the topology for a CRN with six nodes, along with the sets of idle channels for each node. Figure 4(b), shows the bipartite graph constructed by CR $A$. The set of vertices $\mathcal{A}_{A}$, corresponds to the one-hop neighbors of $A$ plus the node itself, $\mathcal{N}_{A}=\{A, B, C, D, E, F\}$, while the set of vertices $\mathcal{B}_{A}$ corresponds to the set of idle channels $C_{A}=\{1,2,3,4,5,7,10\}$. In Figure 4(c), we 


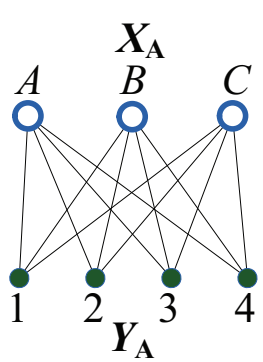

(a)

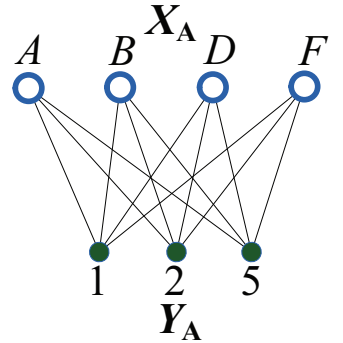

(b)

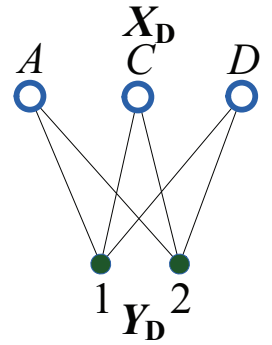

(c)

Figure 5: Bicliques constructed by CRs $A$ and $D$ of figure 4(a), representing possible CR clusters and set of common idle channels.

present the bipartite graph $\mathcal{G}_{D}$ constructed by CR node $D$, for the topology of Figure 4(a). We note that $\mathcal{G}_{A} \neq \mathcal{G}_{D}$ despite the fact that CR nodes $A$ and $D$ are one-hop neighbors. This holds true because, in general, $\mathcal{N}_{A} \neq \mathcal{N}_{D}$ and, under imperfect channel state information, it is expected that $C_{A} \neq C_{D}$.

\subsection{Modeling Clusters as Biclique Graphs}

The purpose of our clustering is to group neighboring nodes with similar sets of idle channels. This criterion improves the bandwidth availability for intra-cluster communications. Nodes within a cluster have more idle channels in common for communication. This property is desirable since in cluster-based architectures, the majority of communications occurs between cluster members. Moreover, such clustering inherently implements cooperative spectrum sensing, since individual sensing observations are combined to a consensus regarding spectrum availability $[7,8,28]$. Note, however, that pairwise communications between cluster members are not restricted to common channels. CRs can use any pairwise shared frequency band to engage in pairwise communications.

To meet our goal, we create clusters where a set $X$ of CR nodes has a set $Y$ of idle channels in common. We model such clusters after a biclique graph $Q(X, Y, E)$. Biclique graphs are special cases of bipartite graphs where all vertices of one side of the bipartition are connected to all vertices of the other side (i.e., they are complete subgraphs of a bipartite graph). In particular, a graph $Q(X, Y, E)$ is called biclique if $\forall x \in X$, and $\forall y \in Y$ there exists an edge between $x$ and $y$, i.e., $E=\{(x, y) \mid \forall x \in X, \forall y \in Y\}$. The edge set $E$ can be completely determined by $X, Y$ and hence, is usually omitted from the biclique notation.

Using the bipartite graph representation, clusters can be formed by finding bicliques that satisfy desired performance criteria. A cluster represented by $Q(X, Y)$, has a size $|X|$ with $|Y|$ channels common to all CRs in $X$. As an example, figures 5(a) and 5(b), show two possible bicliques constructed from the bipartite graph of figure 4(b). The biclique of figure 5(a) represents the clustering of CR nodes $\{A, B, C\}$ with channels $\{1,2,3,4\}$ common to all CRs within the cluster. The biclique of figure 5(b) represents the clustering of CR nodes $\{A, B, D, F\}$ with channels $\{1,2,5\}$ common to all four CRs. Figure 5(c), shows a biclique constructed based on the bipartite graph of CR $D$ (figure 4(c)). We observe that the "quality" of the cluster constructed by $D$ is lower compared to that constructed by $A$, since the cluster of $D$ has fewer members, and fewer idle channels in common.

\section{Clustering Criteria Based on Bicliques}

Based on the mapping of clusters to biclique graphs, we examine three clustering criteria for determining the "quality" of a cluster. These criteria are mapped to three instances of the biclique construction problem 
[29, 30]: (a) the maximum node biclique (MNB) problem, (b) the maximum edge biclique (MEB) problem, and (c) the maximum one-sided edge cardinality biclique (MECB) problem.

The goal of our clustering criteria is to create spectrum-aware clusters which adapt to the heterogeneity of the channel availability. When neighboring CRs have highly correlated sets of idle channels, clusters are decided primarily by the physical topology, but when the sets of idle channels are heterogeneous, a balance is achieved between the cluster size and the set of common idle channels within each cluster.

\subsection{The Maximum Node Biclique (MNB) Criterion}

Under the MNB criterion, the goal is to construct a cluster where the sum of the number of cluster members with the number of common idle channels is maximized. The MNB problem can be stated as follows [31].

Cluster construction under the MNB criterion: Consider bipartite graph $\mathcal{G}_{A}\left(\mathcal{A}_{A} \cup \mathcal{B}_{A}, \mathcal{E}_{A}\right)$, constructed by $\mathrm{CR} A$, where $\mathcal{A}=\mathcal{N}_{A} \cup A$ and $\mathcal{B}_{A}=C_{A}$. Let $\ell_{s}=\left|X_{A}\right|+\left|Y_{A}\right|$ be the sum of vertices of a biclique $Q_{A}\left(X_{A}, Y_{A}\right)$ extracted from $\mathcal{G}_{A}$. Find biclique $Q_{A}^{*}\left(X_{A}, Y_{A}\right)$ such that $Q_{A}^{*}\left(X_{A}, Y_{A}\right)=\arg _{Q} \max \ell_{s}$.

The maximization of $\ell_{S}$ leads, in most cases, to a balance between $\left|X_{A}\right|$ and $\left|Y_{A}\right|$. Moreover, it provides a self-tuning mechanism for dynamically adjusting the cluster membership to the heterogeneity of the sensing observations of the CRs. This is illustrated as follows. If the sets of idle channels $\mathcal{C}_{i}, i \in \mathcal{A}_{A}$ are highly correlated, edges from vertices in $\mathcal{A}_{A}$ will be connected to the same set of vertices in $\mathcal{B}_{A}$. In this case, $Q^{*}$ corresponds to the biclique which tends to maximize $\left|X_{A}\right|$. That is, because neighboring CRs have almost the same sets of idle channels, the "best" cluster is the cluster with the largest size. On the other hand, when the sets of idle channels have low correlation (due to severe channel effects or heterogeneity in the set of PRs heard at each CR), constructing a large size cluster leads to a small set of common idle channels. In this case, selecting only the neighboring CRs with similar sets of idle channels is likely to increase $\ell_{s}$. This is true when the rate of increase of $\left|Y_{A}\right|$ (due to the elimination of low overlapping sets) is larger than the rate of decrease of the cluster size $\left|X_{A}\right|$.

The MNB problem can be solved in polynomial time by a mapping to the maximum independent set problem [31,32]. In our context, we are interested in the analytical evaluation of the existence of bicliques with a particular size and structure in terms of $|X|$ and $|Y|$.

\subsection{The Maximum Edge Biclique (MEB) Criterion}

Under the MEB clustering criterion, the goal is to construct a cluster where the product of the number of cluster members with the number of common idle channels is maximized. The MEB problem can be stated as follows [32, 33].

Cluster construction under the MEB criterion: Consider bipartite graph $\mathcal{G}_{A}\left(\mathcal{A}_{A} \cup \mathcal{B}_{A}, \mathcal{E}_{A}\right)$, constructed by CR $A$, where $\mathcal{A}=\mathcal{N}_{A} \cup A$ and $\mathcal{B}_{A}=C_{A}$. Let $\ell_{p}=\left|X_{A}\right|\left|Y_{A}\right|$ be the number of edges of a biclique $Q_{A}\left(X_{A}, Y_{A}\right)$ extracted from $\mathcal{G}_{A}$. Find biclique $Q_{A}^{*}\left(X_{A}, Y_{A}\right)$ such that $Q_{A}^{*}\left(X_{A}, Y_{A}\right)=\arg _{Q} \max \ell_{p}$.

The MEB criterion exhibits a higher sensitivity to changes in $\left|X_{A}\right|$ and $\left|Y_{A}\right|$ compared to the MNB criterion, and thus, clusters of small size or low availability of common idle channels are avoided. This can be illustrated by the example of figure 6(a), which is a bipartite graph representation of the spectrum availability for a set of CRs $\{A, B, C, D, E, F, G\}$. The maximum size biclique constructed by the MNB criterion is shown in Figure 6(b). In this biclique $\ell_{s}=8$, with seven CRs forming a cluster with one idle 


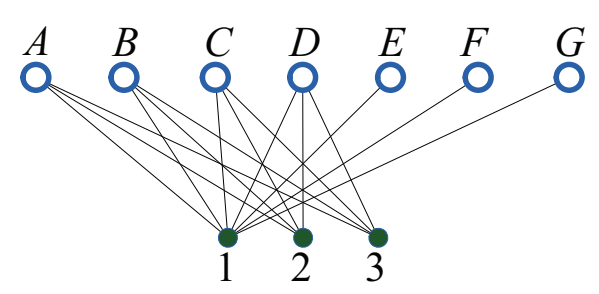

(a)

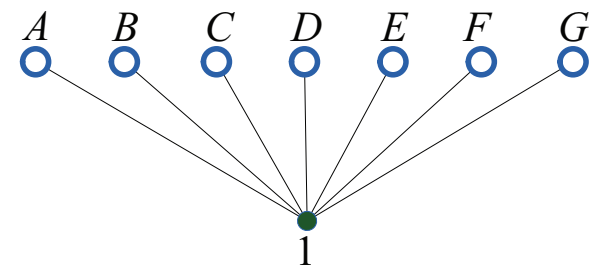

(b)

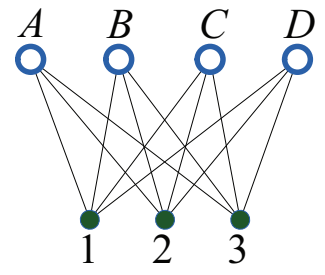

(c)

Figure 6: (a) A realization of a bipartite graph for node $A$, (b) the maximum node biclique, and (c) the maximum edge biclique.

channel (channel 1). Figure 6(c) shows the maximum size biclique constructed by the MEB criterion. In this biclique, CRs $\{A, B, C, D\}$ are clustered, having three idle channels in common.

In this example, the MEB criterion leads to more balanced clustering in terms of the two parameters of interest; the cluster size and the number of common idle channels. This is because the multiplication operation is more sensitive to changes of $|X|$ or $|Y|$ compared to the addition operation. For bivariate function $f_{1}(x, y)=x+y$, the partial derivatives with respect to $x$ and $y$ are equal to 1 , which means that any unit change of $x$ or $y$ can only change $f_{1}$ by one unit. On the other hand, for $f_{2}(x, y)=x y$, the partial derivatives with respect to $x$ and $y$ are equal to $y$ and $x$, respectively. Therefore, a unit change of $x$ or $y$ will change $f_{2}$ by $y \geq 1$ or $x \geq 1$, respectively. The balance property of $f_{2}$ relative to $f_{1}$ can be shown if a simple trade off relationship between $x$ and $y$ is assumed where $1 \leq x, y \leq n$ and $x=n-y$. In this model, a unit of change in one variable causes a unit of change in the other variable and vice versa. For this simple trade off model, $f_{1}$ is maximum for any combination of $x, y$ making a cluster with $|X|=n-1$ and $|Y|=1$ of equal quality to a cluster with $|X|=|Y|=\frac{n}{2}$, On the other hand, $f_{2}$ is maximized only when $x=y=\frac{n}{2}$.

While the MEB criterion is expected to produce better quality clusters compared to the MNB criterion, finding the maximum edge biclique is known to be an NP-complete problem [33]. Several heuristic methods have been proposed that provide desired approximations [32]. These methods can be employed at the individual CRs to derive $Q^{*}$ given $\mathcal{G}$. In our analysis, we are interested in evaluating the possible bicliques obtained under the PR activity and topological models assumed.

\subsection{The Maximum One-sided Edge Cardinality Biclique (MECB) Criterion}

Under the MECB criterion, the goal is to construct clusters of maximum cluster size under a constraint on the number of common idle channels (or vise versa). The MECB problem can be stated as follows.

Cluster construction under the MECB criterion: Consider bipartite graph $\mathcal{G}_{A}\left(\mathcal{A}_{A} \cup \mathcal{B}_{A}, \mathcal{E}_{A}\right)$, constructed by CR $A$, where $\mathcal{A}=\mathcal{N}_{A} \cup A$ and $\mathcal{B}_{A}=C_{A}$. Let $\ell_{c}=\left|X_{A}\right|$ be the size of a biclique $Q_{A}\left(X_{A}, Y_{A}\right)$ extracted from $\mathcal{G}_{A}$. Find biclique $Q_{A}^{*}\left(X_{A}, Y_{A}\right)$ such that $Q_{A}^{*}\left(X_{A}, Y_{A}\right)=\arg _{Q} \max \ell_{c}$, and $\left|Y_{A}\right| \geq \gamma_{0}$, where $\gamma_{0}$ is a positive integer with $1 \leq \gamma_{0} \leq m$, expressing a desired threshold.

The consideration of this constrained maximization problem aims at creating clusters with some minimum guaranteed performance. By selecting a threshold value on the number of common idle channels, a minimum bandwidth availability is guaranteed within every cluster, provided that sufficient idle channels exist. Once this minimum requirement is satisfied, we maximize the cluster size in order to reduce the number of clusters in the CRN. For instance, for the bipartite graph of figure 6(a), setting a threshold $\gamma_{0} \geq 2$ on the number of common idle channels of any cluster, we guarantee that the biclique of figure $6(\mathrm{~b})$ is not a valid cluster. A similar formulation can be derived when the cluster size is constrained to be $\left|X_{A}\right| \geq \gamma_{0}$, 
and the goal is to maximize $\left|Y_{A}\right|$. The problem of finding the maximum one-sided edge cardinality biclique is known to be NP-complete [29], but greedy and probabilistic suboptimal solutions are known [34].

\section{Distributed Clustering based on the Bipartite Graph Representation}

In this section, we present a distributed clustering algorithm that can be used in conjunction with the MNB, MEB and MECB clustering criteria. Note that our goal is not to develop new coordination mechanisms for distributed clustering. A large body of literature is already available in the context of ad hoc networks (e.g., [35-38]). Our goal is to illustrate the practical application of our problem formulation and solution for the purpose of forming spectrum-aware clusters in CRNs.

\subsection{Clustering based on the Distributed Clustering Algorithm}

Basagni et al. proposed the Distributed Clustering Algorithm (DCA) for partitioning an ad hoc network into clusters [35]. In DCA, every node is assigned a weight (a positive real-valued number), indicating its suitability in serving as a clusterhead $(\mathrm{CH})$. The goal of DCA is to assign nodes to neighboring $\mathrm{CHs}$ with the highest weight. The DCA algorithm has been shown to converge after the exchange of a small number of broadcast messages [35].

The DCA algorithm can be modified to take into account our spectrum-aware clustering criteria. In our context, we select the maximum biclique size in terms of sum of nodes $\left(\ell_{s}\right)$, sum of edges $\left(\ell_{p}\right)$ or maximum cardinality $\left(\ell_{c}\right)$ to represent the weight of each node. Note that this weight is a numerical value that is contingent on the formation of a given cluster (biclique) and not an individual node parameter (such as the node's residual energy). Therefore, the performance metric advertised by a node is materialized only if the corresponding cluster is formed. The steps of the proposed distributed clustering mechanism based on DCA are as follows.

Step 1: Every CR $i$ broadcasts its set of idle channels $C_{i}$.

Step 2: Every CR $i$ constructs a bipartite graph $\mathcal{G}_{i}\left(\mathcal{A}_{i} \cup \mathcal{B}_{i}, \mathcal{E}_{i}\right)$ based on $\mathcal{N}_{i}, \mathcal{C}_{i}$, and the sets $\mathcal{C}_{j}, j \in \mathcal{N}_{i}$ received from its neighbors.

Step 3: Every CR $i$ computes the maximum size biclique $Q_{i}^{*}\left(X_{i}, Y_{i}\right)$, according to one of the three clustering criteria (MNB, MEB or MECB). The size of the maximum biclique becomes the weight $w_{i}$ of CR $i$.

Step 4: Every CR $i$ broadcasts $\left\{w_{i}, X_{i}, Y_{i}\right\}$.

Step 5: If $w_{i}>w_{j}, \forall j \in \mathcal{N}_{i}, \mathrm{CR} i$ declares itself as a $\mathrm{CHs}$ by broadcasting a $\mathrm{CH}(i)$ message. If two or more neighboring CRs have the same weight, priority is given to the CR for which the ratio $\frac{|X|}{|Y|}$ is closest to one. Further ties are resolved by considering the CR with the lowest ID.

Step 6: A CR $i$ that has not declared itself as $\mathrm{CH}$, joins the $\mathrm{CH} j, j \in \mathcal{N}_{i}$ with the highest announced weight for which $i \in X_{j}$. CR $i$ broadcasts a join $(i, C H(j))$ message.

Step 7: If a $\mathrm{CR} i$ has not heard a $\mathrm{CH}$ announcement from any of its neighbors, this node waits until all neighboring nodes $j \in \mathcal{N}_{i}$ with $w_{j} \geq w_{i}, i>j$ and $i \in X_{j}$ announce a decision; either they have joined a cluster or declare themselves as CHs. If all neighboring nodes broadcast join messages, CR $i$ declares itself as a $\mathrm{CH}$. Else it joins the cluster of the neighboring $\mathrm{CH}$ with the highest weight.

An example of the application of the DCA-based algorithm on a sample network topology is shown in Figure 7. In Step 1, nodes exchange their idle channel sets $C_{i}$. In Step 2, every CR $i$ individually constructs bipartite graph $\mathcal{G}_{i}\left(\mathcal{A}_{i} \cup \mathcal{B}_{i}, \mathcal{E}_{i}\right)$ and in Step 3, it computes the maximum size biclique $Q_{i}^{*}\left(X_{i}, Y_{i}\right)$, based on its 


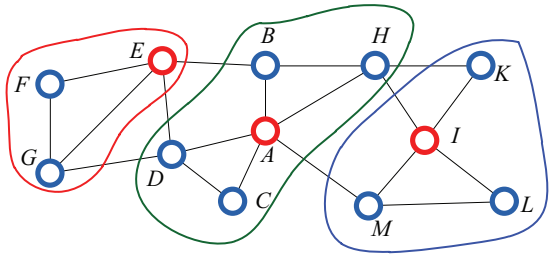

(a) CRN clustering.

\begin{tabular}{c|c|c|c||c|c|c|c}
$\mathrm{CR}$ & $w_{\mathrm{i}}$ & $X_{\mathrm{i}}$ & $Y_{\mathrm{i}}$ & $\mathrm{CR}$ & $w_{\mathrm{i}}$ & $X_{\mathrm{i}}$ & $Y_{\mathrm{i}}$ \\
\hline$A$ & 15 & $\{A, B, C, D, H\}$ & $\{1,2,5\}$ & $G$ & 12 & $\{D, E, F, G\}$ & $\{2,7,9\}$ \\
$B$ & 12 & $\{A, B, E, H\}$ & $\{1,2,5\}$ & $H$ & 8 & $\{A, B, H, I\}$ & $\{2,4\}$ \\
$C$ & 9 & $\{A, C, D\}$ & $\{1,2,5\}$ & $I$ & 15 & $\{H, I, K, L, M\}$ & $\{2,4,8\}$ \\
$D$ & 12 & $\{A, C, \mathrm{E}, \mathrm{F}\}$ & $\{1,2,7\}$ & $K$ & 9 & $\{A, C, \mathrm{E}, \mathrm{F}\}$ & $\{2,4,8\}$ \\
$E$ & 12 & $\{D, E, F, G\}$ & $\{1,2,9\}$ & $L$ & 9 & $\{D, E, F, G\}$ & $\{2,4,8\}$ \\
$F$ & 9 & $\{E, F, G\}$ & $\{1,2,9\}$ & $M$ & 8 & $\{E, F, G\}$ & $\{2,4\}$
\end{tabular}

(b) Table I.

Figure 7: Application of the DCA-based clustering algorithm according to the MNB clustering criterion. Using network topology and idle channel availability information, CRs announce the weights, cluster membership and common idle channels sets shown in Table I. The DCA-based algorithm yields three clusters. CRs $A, E, I$ act as CHs.

view of the topology and spectrum state. In Step 4, each CR announces $\left(w_{i}, X_{i}, Y_{i}\right)$. The list of broadcasts for the topology of Figure 7(a) are shown in Table I of Figure 7(b). Based on the broadcasts of Step 4, CRs $A$ and $I$ have the highest weight among their neighbors $\left(w_{A}=w_{I}=15\right)$ and, therefore, declare themselves as $\mathrm{CHs}$ by broadcasting messages $C H(A)$ and $C H(I)$, respectively. CRs $B, C$, and $D$ join the cluster of $A$, because $A$ is the neighboring $\mathrm{CH}$ with the highest weight and $B, C, D \in X_{A}$. CR $H$ is included in both $X_{A}, X_{I}$ with $w_{A}=w_{I}$. Because $\frac{\left|X_{A}\right|}{\left|Y_{A}\right|}=\frac{\left|X_{I}\right|}{\left|Y_{I}\right|}=1.67, \mathrm{CR} H$ joins the cluster whose $\mathrm{CH}$ has the lowest ID. Therefore, $H$ joins the cluster of $A$. CRs $M, L, K$ join the cluster of $I$. Finally, CR $E$ declares itself as a $C H$ because it has the highest weight among all CRs in $\mathcal{N}_{E}$ that have not yet joined a cluster and nodes $B$ and $D$ have already broadcasted join messages. CRs $F$ and $G$ join the cluster of $E$, because $E$ is the neighboring $\mathrm{CH}$ with the highest weight. The resulting clusters are: $\{E, F, G\}$ with common channels $\{2,7,9\},\{A, B, C, D, H\}$ with common channels $\{1,2,5\}$ and $\{I, K, L, M\}$ with common channels $\{2,4,8\}$.

Communication overhead analysis: Because the clustering algorithm presented in this section is based on DCA, it achieves the same communication efficiency and convergence properties of DCA, as detailed in [35, 39]. The message complexity is in the order of $O\left(n_{C R N}\right)$, where $n_{C R N}$ denotes the CRN size. In particular, in order to complete the clustering process, each node is required to transmit exactly three messages as follows.

- In Step 1, each CR broadcasts one message for communicating its set of idle channels to all its onehop neighbors.

- In Step 4, each CR broadcasts one message for announcing the weight and the corresponding biclique that it individually computed.

- In Steps 5, 6, or 7, each CR broadcasts one message for declaring itself as a CH, or joining a cluster of another neighboring $\mathrm{CH}$.

Adding the cost of all steps yields an overhead of $3 n_{C R N}$ for the entire CRN. Note that the broadcast of the set of idle channel in Step 1 is a requirement of any cooperative sensing mechanism [7, 8, 28], and hence it may not be accounted as overhead of our clustering algorithm.

Note that other clustering methods can be employed to utilize our clustering criteria. As an example, in [12], we proposed a distributed coordination method that makes use of the MEB criterion, called spectrumopportunity clustering (SOC). As opposed to DCA, SOC is a cluster-first algorithm that leads to the creation of clusters before $\mathrm{CHs}$ are elected. The first four steps of SOC are identical to the steps of the DCA-based 
algorithm. This is necessary in order for each CR to obtain the idle channel sets of its one-hop neighbors, and individually construct the biclique of maximum size according to the MNB, MEB or MECB criterion.

\subsection{Re-clustering due to Temporal Variation of Spectrum Availability}

The proposed clustering criteria are based on a snapshot of the spectrum state which is reflected in the sets of idle channels $C_{i}$. These sets vary with time due to the dynamics of PR activity and time-varying phenomena of shadowing and fading [10]. Therefore, clusters created based on one snapshot of the spectrum, are likely to be outdated in the course of time. To account for the temporal variation of spectrum availability, a re-clustering operation is needed. The frequency of the re-clustering varies depending on the dynamics of PR activity. For example, when the PRs are TV stations, the FCC mandates that information on PR activity is updated every few hours [5]. On the other hand, in highly dynamic environments the frequency of re-clustering can lead to excessive communication overhead and clustering instability. To address this problem, we propose an algorithm that applies clustering in a periodic and an on-demand manner. The periodic re-clustering is applied network wide to update the clustering based on long term weight changes. The algorithm for the re-clustering is a clean slate application of the DCA-based clustering algorithm for CRNs, as presented in the previous subsection. The on-demand re-clustering is meant for local rearrangement of CRs into clusters due to temporary changes in channel availability. The steps of the on-demand re-clustering algorithm are as follows.

Step 1: Every CR $i$ broadcasts its set of idle channels $C_{i}$.

Step 2: Every $\mathrm{CH} j$ updates $\left\{w_{j}, X_{j}, Y_{j}\right\}$ for its cluster $j$.

Step 3: If $\left|Y_{j}\right|<\gamma_{0}$, CRs of cluster $j$ change their state to "undecided."

Step 4: A CR $i$ in "undecided" state computes $\left\{w_{i}, X_{i}, Y_{i}\right\}$, by considering only neighboring CRs that are not currently clustered.

Step 5: If $w_{i}$ is larger than the weights of all neighboring CHs, CR $i$ broadcasts $\left\{w_{i}, X_{i}, Y_{i}\right\}$. Else it waits until all "undecided" CRs with lower ID announce their decision.

Step 6: "Undecided" CRs with the highest weight compared to neighboring CHs and other undecided CRs, announce themselves as CHs.

Step 7: A CR $i$ in "undecided" state that has not announced itself as a $\mathrm{CH}$, requests to join the $\mathrm{CH}$ with the maximum weight, assuming that $\left|Y_{j}\right|>\gamma_{0}$ after $i$ joins the cluster of $j$.

Step 8: A CH $j$ confirms the join request of a CR $i$, only if $\left|Y_{j}\right|>\gamma_{0}$ if $i$ is allowed to join cluster $j$. In this case, $\mathrm{CH} j$ broadcasts a granted $(i, \mathrm{CH}(j))$ message. Else $j$ broadcasts a deny $(i, \mathrm{CH}(j))$ message.

Step 9: CR $i$ echoes $j$ 's decision only if the request is accepted. Else, it proceeds with the $\mathrm{CH}$ of the next highest weight.

Step 10: If an "undecided" $\mathrm{CR} i$ has not heard a $\mathrm{CH}$ announcement from any of its neighbors, this node waits until all "undecided" nodes with $w_{i}>w_{j}$ and $i \in X_{j}$, announce their decision. If all neighboring nodes broadcast join or granted messages, $\mathrm{CR} i$ declares itself as a $\mathrm{CH}$. Else it joins the cluster of the neighboring $\mathrm{CH}$ with the highest weight.

Step 11: If a cluster with $|Y| \geq \gamma_{0}$ cannot be formed, "undecided" CRs remain in this state until more channels are freed.

An example of the application of the on-demand re-clustering algorithm for the network of Figure 7(a) is as follows. Assume that $\gamma=2$ and that the set of idle channels for cluster of $\mathrm{CH} A$ has changed to $Y_{A}=\{2\}$, 
because the set of idle channels of $H$ has changed to $C_{H}=\{2,4,8\}$. In Step 1, CH $A$ will obtain the new sets of idle channels from its cluster nodes. It then announces the new values for $\left\{w_{A}, X_{A}, Y_{A}\right\}$. Because $\left|Y_{A}\right|<2$, CRs $\{A, B, C, D, H\}$ update their state to "undecided", while the clusters of CHs $E$ and $I$ remain intact. Every undecided CR computes $\left\{w_{i}, X_{i}, Y_{i}\right\}$. CR $A$ computes a new cluster with $X_{A}=\{A, B, C, D\}$, $Y_{A}=\{1,2,5\}$ and $w_{A}=12$. CRs $B$ and $D$ compute weights lower than the weight of $E$ while CR $C$ computes $X_{C}=\{A, C, D\}, Y_{C}=\{1,2,5\}$ and $w_{C}=9$. Finally, CR $H$ hears $w_{I}=15$ form $I$. Out of the undecided CRs, only $A, C$ announce their weights, since they did not hear a $\mathrm{CH}$ with a higher weight. Based on the weight announcements, CRs $B$ and $D$ decide to join CR $A$ since $w_{A}=w_{E}, \frac{\left|X_{A}\right|}{\left|Y_{A}\right|}=\frac{\left|X_{E}\right|}{\left|Y_{E}\right|}$, but $A>E$. CR $C$ also decides to join $A$. CR $H$ sends a join request to $I$. Because $\left|Y_{I}\right|>2$ after $H$ joins the cluster of $I$, the request of $H$ is accepted. After the on-demand re-clustering the new clusters are $\{E, F, G\}$ with common channels $\{2,7,9\},\{A, B, C, D\}$ with common channels $\{1,2,5\}$, and $\{H, I, K, L, M\}$ with common channels $\{2,4,8\}$.

Communication overhead analysis: Our re-clustering algorithm aims at reducing the communication overhead associated with maintaining clusters up-to-date with the spectrum availability. This is achieved by incorporating the following strategies. First, the composition of a cluster is maintained as long as a minimum number of idle channels remains common to all cluster members. Hence, frequent re-clustering is avoided at the expense of sub-optimal clusters in terms of biclique size (due to the temporal variation of the spectrum). The network-wide periodic re-clustering reconfigures the network to optimal clusters for longer term changes. Second, when a cluster does not meet the minimum requirements in terms of common idle channels, re-clustering is performed locally for the members of that cluster. In the example of Figure 7, only the members of cluster $A$ had to be re-assigned. Moreover, "undecided" CRs are allowed to join neighboring clusters, only if they are not forcing the neighboring cluster to a re-clustering. That is, the share sufficient idle channels with the cluster they join. This design prevents a cascading effect of re-clustering operations, thus limiting the communication overhead and delay until new clusters are formed.

The exact overhead of the re-clustering algorithm is topology and PRN activity dependent. For a single cluster of size $|X|$ whose members need to be re-clustered, we analyze the number of messages that need to be exchanged. Assume that every cluster member in "undecided" state is a neighbor of $\phi$ other CHs. Note that based on DCA [39], no two CHs can be neighbors and, hence $\phi<6$ (simple geometric constructions can show that a node cannot have more than 5 independent neighbors). The re-clustering communication overhead is as follows.

- In Step 1, each CR broadcasts its set of idle channels ${ }^{3}$ accounting for a total of $|X|$ messages.

- In Step 2, every neighboring $\mathrm{CH}$ announces its weight and biclique structure, accounting for $\phi$ messages.

- In Steps 5 and 6, the worst case scenario in terms of overhead is realized when none of the "undecided" CRs becomes a $\mathrm{CH}$.

- In Step 7, an "undecided" CR can request to join up to $\phi$ pre-existing clusters, yielding a message overhead up to $\phi|X|$ messages.

- In Step 8, each of the $\phi$ neighboring $\mathrm{CHs}$ replies in up to $|X|$ join requests, totaling $\phi|X|$ messages.

- In Step 10, "undecided" CRs that did not join any neighboring pre-existing cluster announce themselves as $\mathrm{CH}$ or join the cluster of another "undecided" CR, yielding up to $|X|$ messages.

\footnotetext{
${ }^{3}$ Broadcasting of the set of idle channels is a periodic function required by the spectrum sensing process [7-9, 28, 40].
} 


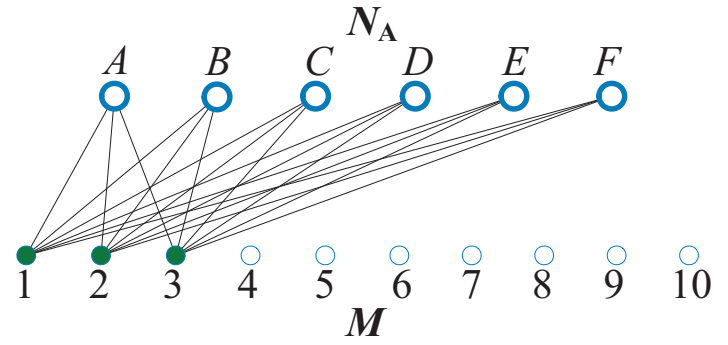

(a)

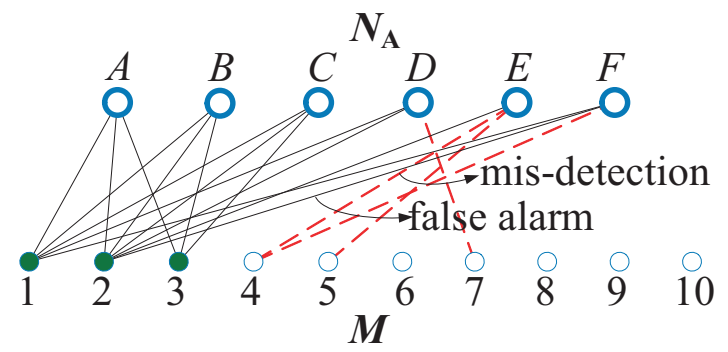

(c)

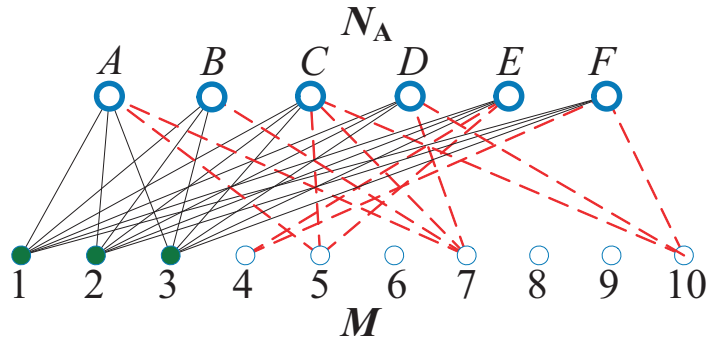

(b)

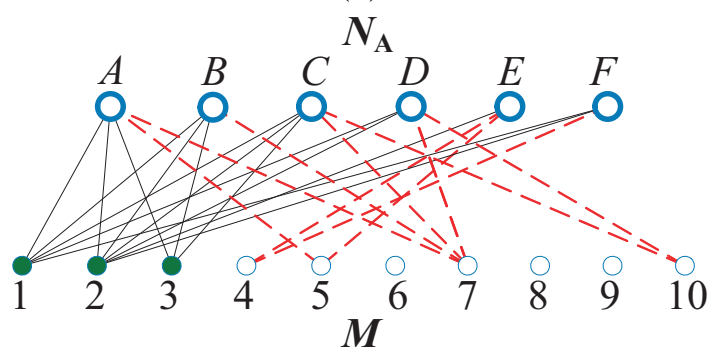

(d)

Figure 8: (a) A bipartite graph for a topology of type-I under perfect channel state information. All CRs are connected to the set of idle channels $\{1,2,3\}$, (b) a bipartite graph for a topology of type-II under perfect channel state information. The dashed lines indicate CRs that are out of range from PRs that are active on a given channel, (c) a bipartite graph for topologies of type-I under imperfect channel state information. Events of false alarm and mis-detection prevent the correct estimation of the channel state (missing solid lines to idle channels indicate events of false alarm, and dashed lines indicate mis-detection of occupied channels), (d) a bipartite graph for topologies of type-II under imperfect channel state information. A combination of the bipartite graphs in (b) and (c).

Adding the number of messages transmitted at each step yields a communication overhead which is upper bounded by $(2+2 \phi)|X|+\phi \leq 12|X|+5$ messages.

\section{Performance Evaluation}

In this section, we analytically compute the performance of the proposed clustering criteria. We are focusing on determining the quality of clusters that are constructed under the graph-based representation of the network topology and spectrum availability. We validate our theoretical results by performing extensive simulations.

\subsection{Bipartite Graph Models}

We first analyze the impact of the topology and channel state information models on the bipartite graph structure. This structure is employed in the analytical evaluation of the clustering performance.

Perfect Channel State Information. Consider the bipartite graph $\mathcal{G}_{x}\left(\mathcal{A}_{x} \cup \mathcal{B}_{x}, \mathcal{E}_{x}\right)$ constructed by a CR $x$ under perfect channel state information. For $\mathcal{G}_{x}$, any vertex $i \in \mathcal{C}_{x}$ corresponding to an idle channel is connected to all vertices in $\mathcal{A}_{x}$. Hence, edges corresponding to idle channels appear in groups of size $\left(\left|\mathcal{N}_{x}\right|+1\right)$.

For type-I topologies, no other edges exist in $\mathcal{G}_{x}$. Figure 8(a) shows the structure of a bipartite graph constructed by $A$, for the CRN of Figure 4(a), when channels $\{1,2,3\}$ are assumed to be idle The vertices 
corresponding to channels $\{1,2,3\}$ are connected to all vertices in $\mathcal{A}_{x}$. For type-II topologies, the structure of $\mathcal{G}_{x}$ depends on the positions of the PRs relative to CR $x$ and its one-hop neighbors. Based on the model in Section 2, a PR is within a range of $x$ with probability $p_{w}$ (see eq. (4)). Therefore, an edge between a vertex $i$ corresponding to an occupied channel and $x$ exists with probability $p_{w}$. Moreover, for the same vertex $i$, an edge exists between $i$ and a CR $y \in \mathcal{N}_{x}$, if $y$ is not in the range of the PR occupying $i$. According to equation (5), this event occurs with probability $p_{c}$. Figure $8(\mathrm{~b})$ shows a bipartite graph constructed for typeII topologies, under perfect channel state information. Note that a subset of vertices in $C_{A}$ are connected to all vertices in $A \cup \mathcal{N}_{A}$. These vertices correspond to the idle channels. Additional edges exist between $A \cup \mathcal{N}_{A}$ and occupied channels.

Imperfect Channel State Information. When sensing information is not obtained from a database, CRs are subject to imperfect channel sensing. In this case, the edges of the bipartite graph jointly depend on the PR activity and the probabilities of mis-detection and false alarm. Let's consider topologies of type-I. For a bipartite graph $\mathcal{G}_{x}$, if a channel $i$ is idle, the corresponding vertex is connected to a vertex $j \in \mathcal{A}_{x}$ with probability $\left(1-p_{f}\right)$. Furthermore, a vertex $i$ corresponding to an occupied channel $i$, is connected to a vertex $j \in \mathcal{A}_{x}$, with probability $p_{m}$. This is because all CRs sense the same set of PRs. In Figure 8(c), we show one realization of a bipartite graph constructed under the imperfect sensing model for type-I topologies. Edges connecting CRs to idle channels independently exist with probability $\left(1-p_{f}\right)$, while edges corresponding to occupied channels independently exist with probability $p_{m}$.

For type-II topologies, edges connecting CRs to idle channels exist with the same probability as in the type-I case (a.k.a., $\left.\left(1-p_{f}\right)\right)$. On the other hand, for occupied channels, the position of the active PRs relative to $x$ must be considered. An edge between an occupied channel $i$ and $x$ exists the following probability.

$$
\begin{aligned}
\operatorname{Pr}[(i, x)=1] & =\operatorname{Pr}\left[d_{x y} \leq R, i \text { mis-detected } \vee d_{x y}>R, i \text { no false alarm }\right] \\
& =\operatorname{Pr}\left[d_{x y} \leq R, i \text { mis-detected }\right]+\operatorname{Pr}\left[d_{x y}>R, i \text { no false alarm }\right] \\
& =\operatorname{Pr}\left[d_{x y} \leq R\right] \operatorname{Pr}[i \text { mis-detected }]+\operatorname{Pr}\left[d_{x y}>R\right] \operatorname{Pr}[i \text { no false alarm }] \\
& =\left(1-p_{w}\right) p_{m}+p_{w}\left(1-p_{f}\right) .
\end{aligned}
$$

In equation (6a), we considered the probability that CR $x$ is within range of the PR that is active on channel $i$ but $i$ is mis-detected, or that $x$ is out of range from the active PR and the state of channel $i$ is correctly estimated. Equation (6b) follows from the fact that $d_{x y} \leq R$ and $d_{x y}>R$ are mutually exclusive events. Equations (6c) and (6d) follow by the assumption that the events of mis-detection and false alarm are assumed independent of the distance between $x$ and $y$. Note that the perfect channel state model can be considered as a special case of the imperfect one. The probability of edge existence for occupied channels under the perfect channel state model can be obtained from (6d) by setting $p_{m}=p_{f}=0$.

Similarly, one can show that an edge connects the vertex corresponding to occupied channel $i$ to a neighbor $j \in \mathcal{N}_{x}$ with probability $\left(1-p_{c}\right) p_{m}+p_{c}\left(1-p_{f}\right)$. In Figure 8(d), we show one realization of a bipartite graph constructed under the imperfect sensing model, for type-II topologies. In this example, channels $\{1,2,3\}$ are not occupied by PRs. Edges from vertices $\{1,2,3\}$ are independently connected to vertices in the other side of the bipartition with probability $\left(1-p_{f}\right)$. Channels $\{4-10\}$ are assumed to be occupied by PRs. Edges from vertices $\{4-10\}$ are connected to vertex $A$ with probability $\left(1-p_{w}\right) p_{m}+p_{w}\left(1-p_{f}\right)$, and all other nodes with probability $\left(1-p_{c}\right) p_{m}+p_{c}\left(1-p_{f}\right)$.

\subsection{Evaluation Metrics}

In this section, we define the metrics used in our performance evaluation. Our goal is to evaluate the existence of clusters of different "quality" (biclique size) under the different sensing, topology, and activity 
models.

Let $Z_{\ell}$ denote the number of bicliques of "size" $\ell$. The variable $Z_{\ell}$ is defined as,

$$
Z_{\ell} \sum_{X \subseteq \mathcal{A}, Y \subseteq \mathcal{B}, \operatorname{size}(Q(X, Y))=\ell,|X|,|Y| \geq 1} \mathrm{I}_{(X, Y)},
$$

where the indicator function $\mathrm{I}_{(X, Y)}$ is defined as,

$$
\mathrm{I}_{(X, Y)}= \begin{cases}1, & \text { if } \operatorname{size}(Q(X, Y))=\ell \\ 0, & \text { otherwise }\end{cases}
$$

In (7), we consider all possible values of $|X|$ and $|Y|$ that yield a biclique of a size $\ell$. By definition, the expected number of bicliques $\mathrm{E}\left[Z_{\ell}\right]$ of size $\ell$ is,

$$
\begin{aligned}
\mathrm{E}\left[Z_{\ell}\right] & =\mathrm{E}\left[\sum_{X \subseteq \mathcal{A}, Y \subseteq \mathcal{B}, \operatorname{size}(Q(X, Y))=\ell,|X|,|Y| \geq 1} \mathrm{I}_{(X, Y)}\right] \\
= & \sum_{X \subseteq \mathcal{A}, Y \subseteq \mathcal{B}, \operatorname{size}(Q(X, Y))=\ell,|X|,|Y| \geq 1} \mathrm{E}\left[\mathrm{I}_{(X, Y)}\right] \\
= & \sum_{X \subseteq \mathcal{A}, Y \subseteq \mathcal{B}, \operatorname{size}(Q(X, Y))=\ell,|X|,|Y| \geq 1} \operatorname{Pr}\left[\mathrm{I}_{(X, Y)}=1\right] .
\end{aligned}
$$

The expectation of $Z_{\ell}$ is used to analytically evaluate the probability of existence a biclique of size $\ell$. We employ the Chebyshev inequality to derive a relationship between the probability of existence of a biclique of size $\ell$, and the first and second statistical moments of $Z_{\ell}$. For $s>0$, the Chebyshev inequality states,

$$
\operatorname{Pr}\left[\left|Z_{\ell}-\mathrm{E}\left[Z_{\ell}\right]\right| \geq s\right] \leq \operatorname{Var}\left[Z_{\ell}\right] / s^{2} .
$$

For $\mathrm{E}\left[Z_{\ell}\right]>1$, setting $s=\mathrm{E}\left[Z_{\ell}\right]-1$ yields,

$$
\begin{aligned}
\operatorname{Pr}\left[Z_{\ell} \geq 1\right] & \geq \operatorname{Pr}\left[1 \leq Z_{\ell} \leq 2 \mathrm{E}\left[Z_{\ell}\right]-1\right] \\
& =\operatorname{Pr}\left[-s+\mathrm{E}\left[Z_{\ell}\right] \leq Z_{\ell} \leq s+\mathrm{E}\left[Z_{\ell}\right]\right] \\
& =\operatorname{Pr}\left[\left|Z_{\ell}-\mathrm{E}\left[Z_{\ell}\right]\right| \leq s\right] \\
& =1-\operatorname{Pr}\left[\left|Z_{\ell}-\mathrm{E}\left[Z_{\ell}\right]\right| \geq s\right] \\
& \geq 1-\operatorname{Var}\left[Z_{\ell}\right] /\left(\mathrm{E}\left[Z_{\ell}\right]-1\right)^{2} .
\end{aligned}
$$

For values of $\operatorname{Var}\left[Z_{\ell}\right] /\left(\mathrm{E}\left[Z_{\ell}\right]-1\right)^{2} \leq 1$, the Chebyshev inequality yields a lower bound on the probability of existence of bicliques of size $\ell$. This yields the biclique sizes that are likely to occur, given the PR activity, sensing and topological models.

We also evaluate the probability of obtaining bicliques that satisfy desired constraints on $|X|$ and $|Y|$. As an example, the average number of bicliques of size $\ell$, with $|X| \geq x$ and $|Y| \geq y$ is defined as,

$$
\mathrm{E}\left[Z_{\ell}|| X|\geq x,| Y \mid \geq y\right]=\sum_{X \subseteq \mathcal{A}, Y \subseteq \mathcal{B}, \operatorname{size}(Q(X, Y))=\ell,|X| \geq x,|Y| \geq y,|Y|=\ell-x,|X|} \operatorname{Pr}\left[\mathrm{I}_{(X, Y)}=1\right] .
$$


Similarly, we bound

$$
\operatorname{Pr}\left[Z_{\ell} \geq 1|| X|\geq x,| Y \mid \geq y\right] \geq 1-\frac{\operatorname{Var}\left[Z_{\ell}|| X|\geq x,| Y \mid \geq y\right]}{\left(\mathrm{E}\left[Z_{\ell}|| X|\geq x,| Y \mid \geq y\right]-1\right)^{2}},
$$

for $\mathrm{E}\left[Z_{\ell}|| X|\geq x| Y \mid, \geq y\right]>1$. We now analytically evaluate the clustering performance of the MNB, $\mathrm{MEB}$, and MECB clustering criteria.

\subsection{The MNB Criterion.}

Under the MNB criterion, the cluster quality is characterized by the sum $\ell_{s}=|X|+|Y|$. In this section, we analytically evaluate the expected number of clusters (bicliques) with a particular value $\ell_{s}$, for each of the PR activity and channel state information models.

Proposition 1. The expected number $E\left[Z_{\ell_{s}}\right]$ of clusters with value $\ell_{s}=|X|+|Y|$ is given by,

$$
E\left[Z_{\ell_{s}}\right]=\sum_{i=\max \left\{1, \ell_{s}-n\right\}}^{\min \left\{m, \ell_{s}-1\right\}} \operatorname{Pr}[N=i] \sum_{\alpha=0}^{\min \left\{i, \ell_{s}-1\right\}} \sum_{\beta=\max \left\{0, \ell_{s}-n-\alpha\right\}}^{\min \left\{\ell_{s}-1-\alpha, m-i\right\}} C\left(n, \ell_{s}-\alpha-\beta\right) C(i, \alpha) C(m-i, \beta) \Pi_{S}^{t}\left(\ell_{s}, \alpha, \beta\right) .
$$

Recall, that $C(n, k)=\left(\begin{array}{l}n \\ k\end{array}\right)$. Here, the probability $\Pi_{S}^{t}(\alpha, \beta)$ of the appearance of a biclique of size $\ell_{s}$ depends on the PR activity and channel state information models.

$$
\Pi_{S}^{t}\left(\ell_{s}, \alpha, \beta\right)=\left\{\begin{array}{l}
1, \quad(i), \\
p_{w}^{\beta} p_{c}^{\beta\left(\ell_{s}-\alpha-\beta-1\right)}, \quad(i i), \\
\left(1-p_{f}\right)^{\left(\ell_{s}-\alpha-\beta\right) \alpha} p_{m}^{\left(\ell_{s}-\alpha-\beta\right) \beta}, \quad(\text { iii }), \\
\left.\left(1-p_{f}\right)^{\left(\ell_{s}-\alpha-\beta\right) \alpha}\left(\left(1-p_{w}\right) p_{m}+p_{w}\left(1-p_{f}\right)\right)^{\beta}\left(\left(1-p_{c}\right) p_{m}+p_{c}\left(1-p_{f}\right)\right]\right)^{\beta\left(\ell_{s}-\alpha-\beta-1\right)},(i v),
\end{array}\right.
$$

for (i) perfect channel state information, type-I topologies, (ii) perfect channel state information, type-II topologies, (iii) imperfect channel state information, type-I topologies, and (iv) imperfect channel state information, type-II topologies, respectively.

Proof. The proof of proposition 1 is provided in Appendix B.

In proposition 1 , the value of $\Pi_{S}^{t}\left(\ell_{s}, \alpha, \beta\right)$ for case (iv), represents the most general scenario. From (iv), all other cases can be derived by setting parameters to appropriate values. Setting $p_{f}=p_{m}=p_{c}=p_{w}=0$ yields case (i), setting $p_{f}=p_{m}=0$ yields case (ii), and setting $p_{c}=p_{w}=0$, yields case (iii). From proposition 1 , we can also evaluate $\mathrm{E}\left[Z_{\ell_{s}}\right]$ for any desired PR activity model. For instance, when the PR activity follows the binomial model in (2),

$\mathrm{E}\left[Z_{\ell_{s}}\right]=\sum_{i=\max \left\{1, \ell_{s}-n\right\}}^{\min \left\{m, \ell_{s}-1\right\}} C(m, i) p^{i}(1-p)^{m-i} \sum_{\alpha=0}^{\min \left\{i, \ell_{s}-1\right\}} \sum_{\beta=\max \left\{0, \ell_{s}-n-\alpha\right\}}^{\min \left\{\ell_{s}-1-\alpha, m-i\right\}} C\left(n, \ell_{s}-\alpha-\beta\right) C(i, \alpha) C(m-i, \beta) \Pi_{S}^{t}\left(\ell_{s}, \alpha, \beta\right)$.

For the Poisson model in (3),

$$
\mathrm{E}\left[Z_{\ell_{s}}\right]=\sum_{i=\max \left\{1, \ell_{s}-n\right\}}^{\min \left\{m, \ell_{s}-1\right\}} \frac{\frac{\rho^{m-i}}{(m-i) !}}{\sum_{j=0}^{m} \frac{\rho^{j}}{j !}} \sum_{\alpha=0}^{\min \left\{i, \ell_{s}-1\right\}} \sum_{\beta=\max \left\{0, \ell_{s}-n-\alpha\right\}}^{\min \left\{\ell_{s}-1-\alpha, m-i\right\}} C\left(n, \ell_{s}-\alpha-\beta\right) C(i, \alpha) C(m-i, \beta) \Pi_{S}^{t}\left(\ell_{s}, \alpha, \beta\right) .
$$


Under perfect channel state information and for type-I topologies, the bipartite graph representing the network topology and spectrum availability obtains a simple form that can be further exploited to analytically evaluate the probability distribution of the largest size biclique, the distribution of the cluster size $|X|$, and the distribution of the number of common idle channels $|Y|$. These are expressed in the following lemmas.

Lemma 1. Let $\mathcal{L}_{\max }$ be a discrete random variable expressing the value of $\ell_{s}$ for a cluster which maximizes $\ell_{s}$. Under perfect channel state information and for topologies of type-I, $\mathcal{L}_{\max } \sim N$, i.e., $\mathcal{L}_{\max }$, is identically distributed to the number of idle channels in the system.

Proof. Consider a bipartite graph $\mathcal{G}(\mathcal{A} \cup \mathcal{B}, \mathcal{E})$ constructed under perfect channel state information, and for type-I topologies. The graph $\mathcal{G}$ is itself a biclique, because vertices corresponding to idle channels are connected to all vertices corresponding to CRs and no other edges are present in the bipartite graph. In fact, $\mathcal{G}$ is the biclique of maximum size, since it contains all vertices of $\mathcal{G}$. The maximum size can be expressed as a random variable $\mathcal{L}_{\max }$, which depends on the number of idle channels and obtains discrete values from the alphabet $\{0, n+1, n+2, \ldots, n+m\}$. $\mathcal{L}_{\max }=0$ when no idle channels exist ( $\mathcal{G}$ has no edges). This occurs with probability $\operatorname{Pr}[\mathrm{N}=0]$. If $i>0$, then $\mathcal{L}_{\max }=n+i$ with probability $\operatorname{Pr}[\mathrm{N}=i]$. Combining both cases yields,

$$
\operatorname{Pr}\left[\mathcal{L}_{\max }=\ell_{s}\right]= \begin{cases}\operatorname{Pr}[\mathrm{N}=0], & \ell_{s}=0 \\ \operatorname{Pr}[\mathrm{N}=i], & \ell_{s}=n+i, 1 \leq i \leq m,\end{cases}
$$

which is the probability mass function of $\mathrm{N}$.

Lemma 2. Under perfect channel state information, and for type-I topologies, the cluster size $|X|$ of the cluster with maximum value of $\ell_{s}$ follows a Bernoulli distribution with parameter $1-\operatorname{Pr}[N=0]$, i.e.,

$$
\operatorname{Pr}[|X|=x]= \begin{cases}\operatorname{Pr}[N=0], & x=0 \\ 1-\operatorname{Pr}[N=0], & x=m .\end{cases}
$$

Lemma 3. Under perfect channel state information and for type-I topologies, the number of common idle channels $|Y|$, in the cluster with maximum value of $\ell_{s}$ is identically distributed to the number of idle channels, i.e., $|Y| \sim N$.

The proofs of Lemmas 2, 3 follow in a straightforward manner from the properties of the bipartite graph under perfect channel state information. Note that Lemmas 2 and 3 illustrate the versatility of our clustering criteria in adjusting to the PR activity and sensing conditions. They state that when at least one channel is idle, the MNB criterion yields a cluster of maximum size, equal to the neighborhood size of each CR. This is because under perfect channel state information, all CRs have identical sets of idle channels and hence, spectrum availability is not factoring into cluster memberships. Instead, the clusters are decided by topological criteria, similarly to ad hoc networks with fixed spectrum. On the other hand, when the sets of idle channels vary from CR to CR, the cluster size is adjusted to allow for higher overlap in the set of common idle channels within each cluster.

Evaluating the probability distributions for $\mathcal{L}_{\max },|X|,|Y|$, under imperfect channel state information involves complex summation formulas. Instead, we use the Markov and Chebyshev inequalities, as defined in section 6.2 to characterize the probability of existence for bicliques of a given size. Here, based on the computation of $\mathrm{E}\left[Z_{\ell}\right]$ on proposition 1 , the respective probability $\operatorname{Pr}\left[Z_{\ell} \geq 1\right]$ is bounded by this inequality. Note that the Chebyshev bound requires the computation of the variance of $Z_{\ell_{s}}$. Due to the complexity of the calculations, a numerical computation of the variance is preferred. This is feasible because the expected 
neighborhood sizes $N_{i}$ and number of available channels $|\mathcal{M}|$ are anticipated to be relatively small (in the order of tens of nodes and channels).

\subsection{The MEB Criterion}

Under the MEB criterion, the cluster quality is characterized by the product $\ell_{p}=|X|+|Y|$. Similarly to the case of the MNB criterion, we analytically evaluate the expected number of clusters (bicliques) with a particular value $\ell_{p}$, for each of the PR activity and channel state information models.

Proposition 2. The expected number $E\left[Z_{\ell_{p}}\right]$ of clusters with value $\ell_{p}=|X||Y|$, under perfect channel state information and for type-I topologies is given by,

$$
E\left[Z_{\ell_{p}}\right]=\sum_{i=\max \left\{1,\left[\frac{\ell_{p}}{n}\right]\right\}}^{\min \left\{m, \ell_{p}\right\}} \operatorname{Pr}[N=i] \sum_{\left.j=\max \left\{1, \frac{\ell_{p}}{n}\right]\right\}, \frac{\ell_{p}}{j} \in \mathbb{Z}^{+}}^{\min \left\{i, \ell_{p}\right\}} C\left(n, \frac{\ell_{p}}{j}\right) C(i, j),
$$

where $\mathbb{Z}^{+}$denotes the set of positive integers.

The proof of proposition 2 is provided in Appendix C. The steps of the proof are similar to the proof of proposition 1. While the objective of the MEB criterion differs from the objective of the MNB criterion, the clustering performance of both criteria is expected to be the same under perfect channel state information. That is, the probability distribution of $|X|$ and $|Y|$ for clusters constructed under the MEB criterion is given by Lemmas 2, 3. This is because under a perfect channel state information model, the bipartite graph constructed by CRs forms a biclique of maximum sum of vertices and of maximum product of edges (concurrent maximization of $|X|$ and $|Y|$ maximizes both $(|X|+|Y|)$ and $|X||Y|)$. Hence, the MNB, MEB criteria yield the same clusters.

Proposition 3. The expected number $E\left[Z_{\ell_{p}}\right]$ of clusters with value $\ell_{p}=|X \| Y|$ is given by:

$$
E\left[Z_{\ell_{p}}\right]=\sum_{i=0}^{\min \left\{m, \ell_{p}\right\}} \operatorname{Pr}[N=i] \sum_{\alpha=0}^{i} \sum_{\beta=0}^{m-i} C\left(n, \frac{\ell_{p}}{\alpha+\beta}\right) C(i, \alpha) C(m-i, \beta) \Pi_{P}^{t}\left(\ell_{p}, \alpha, \beta\right),
$$

with $\left\lceil\frac{\ell_{p}}{n}\right\rceil \leq \alpha+\beta \leq \ell_{p}$ and $\frac{\ell_{p}}{\alpha+\beta} \in \mathbb{Z}^{+}$. Here the probability $\Pi_{P}^{t}\left(\ell_{p}, \alpha, \beta\right)$ of the appearance of a biclique of size $\ell_{p}$ depends on the type:

$$
\Pi_{P}^{t}(\alpha, \beta)=\left\{\begin{array}{l}
p_{w}^{\beta} p_{c}^{\beta\left(\frac{\ell_{p}}{\alpha+\beta}-1\right)}, \quad(i), \\
\left(1-p_{f}\right)^{\frac{\alpha \ell_{p}}{\alpha+\beta}} p_{m}^{\frac{\beta \ell_{p}}{\alpha+\beta}}, \quad(i i), \\
\left(1-p_{f}\right)^{\frac{\alpha \ell_{p}}{\alpha+\beta}}\left(\left(1-p_{w}\right) p_{m}+p_{w}\left(1-p_{f}\right)\right)^{\beta}\left(\left(1-p_{c}\right) p_{m}+p_{c}\left(1-p_{f}\right)\right)^{\beta\left(\frac{\ell_{p}}{\alpha+\beta}-1\right)} \quad(i i i),
\end{array}\right.
$$

for (i) perfect channel state information, type-II topologies, (ii) imperfect channel state information, type-I topologies, and (iii) imperfect channel state information, type-II topologies, respectively.

The proof is similar to the proof of proposition 1 and hence, is omitted. From propositions 2 and 3, the probability of constructing a cluster with a particular value of $\ell_{p}$ is derived based on the Chebyshev inequality. 


\subsection{The MECB Criterion}

Under the MECB criterion, the cluster quality is characterized by the size $\ell_{c}=|X|\left(\ell_{c}=|Y|\right)$ of the cluster, under a constraint $|Y| \geq \gamma_{0}\left(|X| \geq \gamma_{0}\right)$, on the common set of idle channels (cluster size). In this section, we analytically evaluate the expected number of clusters (bicliques) with a particular value $\ell_{c}$, for each of the PR activity and channel state information models.

Perfect channel state information, Type-I topologies. When perfect channel state information is available, $\mathrm{E}\left[Z_{\ell_{c}}\right]$ is expressed by the following proposition.

Proposition 4. The expected number of clusters of size $\ell_{c}=|X|$ with $|Y| \geq \gamma_{0}$, for topologies of type-I is given by,

$$
E\left[Z_{\ell_{c}}\right]=C\left(n, \ell_{c}\right) \sum_{i=\gamma_{0}}^{m} \operatorname{Pr}[N=i] .
$$

Proof. It follows immediately by noting that when perfect channel state information is available, the bipartite graph constructed by any CR is a biclique. When $|Y| \geq \gamma_{0}$ channels are idle, the corresponding vertices in $Y$ are connected to all vertices in $X$. Hence, there are a total of $C\left(n, \ell_{c}\right)$ distinct bicliques of size $\ell_{c}$. That is, there are $C\left(n, \ell_{c}\right)$ possible clusters with size $\ell_{c}$.

Other scenarios. For all other combination of channel state information and topology models, $\mathrm{E}\left[Z_{\ell_{c}}\right]$ is expressed by the following proposition:

Proposition 5. The expected number of clusters of size $\ell_{c}=|X|$, with $|Y| \geq \gamma_{0}$ is given by,

$$
E\left[Z_{\ell_{s}}\right]=\sum_{i=0}^{m} \operatorname{Pr}[N=i] \sum_{\alpha=0}^{m} \sum_{\beta=\max \left\{0, \gamma_{0}-\alpha-i\right\}}^{m-i} C\left(n, \ell_{c}\right) C(i, \alpha) C(m-i, \beta) \Pi_{C}^{t}\left(\ell_{c}, \alpha, \beta\right) .
$$

Here the probability $\Pi_{C}^{t}\left(\ell_{c}, \alpha, \beta\right)$ of the appearance of a biclique of size $\ell_{c}$ depends on the type:

$$
\Pi_{C}^{t}\left(\ell_{c}, \alpha, \beta\right)=\left\{\begin{array}{l}
p_{w}^{\beta} p_{c}^{\beta\left(\ell_{c}-1\right)}, \quad(i), \\
\left(1-p_{f}\right)^{\alpha \ell_{c}} p_{m}^{\beta \ell_{c}}, \quad(i i), \\
\left(\left(1-p_{w}\right) p_{m}+p_{w}\left(1-p_{f}\right)\right)^{\beta}\left(\left(1-p_{c}\right) p_{m}+p_{c}\left(1-p_{f}\right)\right)^{\beta\left(\ell_{c}-1\right)}, \quad \text { (iii), }
\end{array}\right.
$$

for (i) perfect channel state information, type-II topologies, (ii) perfect channel state information, type-I topologies, and (iii) imperfect channel state information, type-II topologies, respectively.

Proof. The proof is similar to the proof of proposition 1, and hence is omitted.

\subsection{Performance Evaluation via Simulations}

In this section, we verify the validity of our theoretical results and study the performance of the proposed clustering criteria via simulations. In our simulation setup, we considered a CRN co-existing with a PRN which was licensed to use a set of $m=10$ frequency bands. The licensed bands were occupied by PRs according to the binomial model in (2) (similar results can be derived for the Poisson PR activity model in (3)). For type-I topologies, we considered a single-hop CR neighborhood of size $n=10$, affected by the PR activity of 10 PRs. In each simulation run, a set of channels was occupied by PRs leading to the construction of a bipartite graph. Using this bipartite graph, the set of possible cluster formations were computed for each clustering criterion. The experiments were repeated 10,000 times for each set of parameters, in order to evaluate the average performance. 


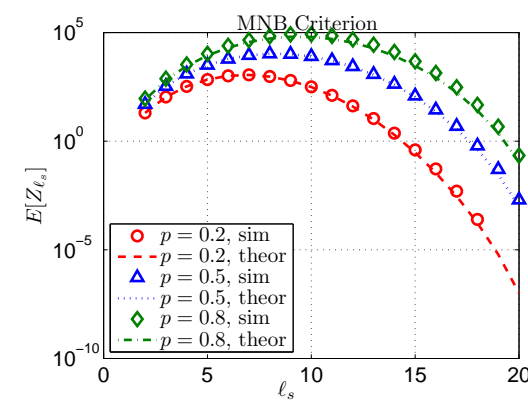

(a)

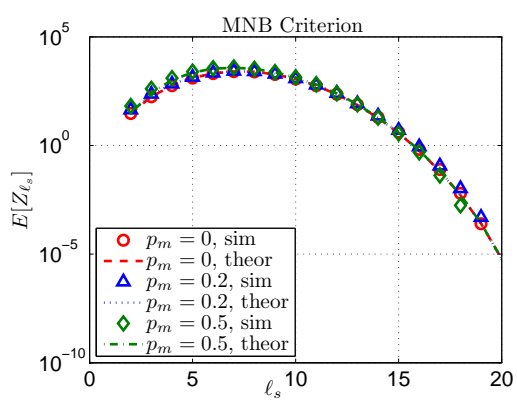

(d)

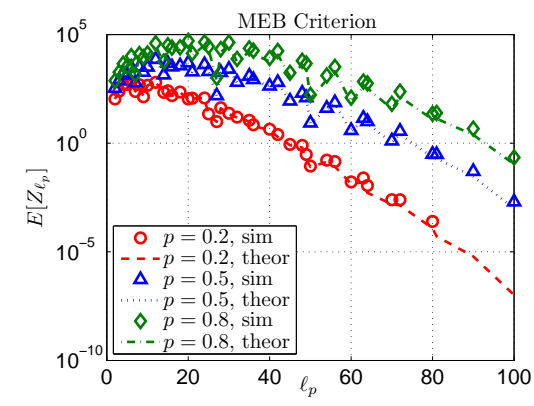

(b)

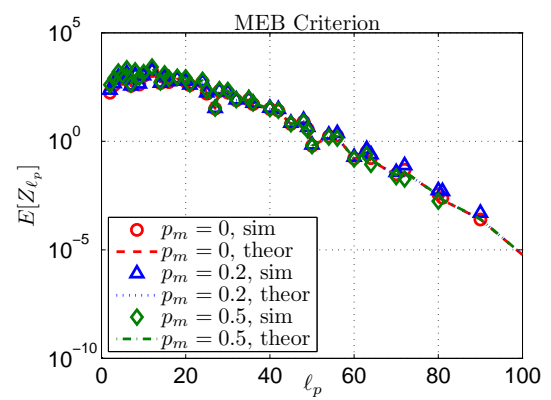

(e)

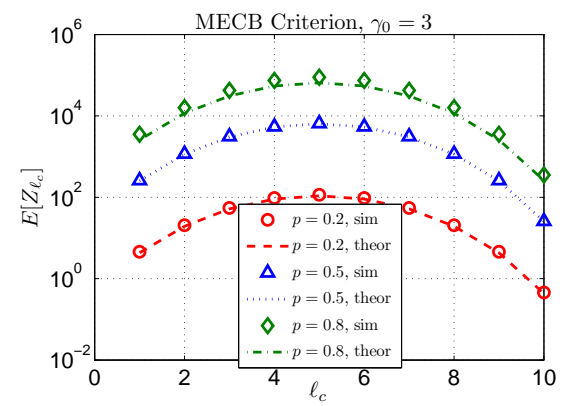

(c)

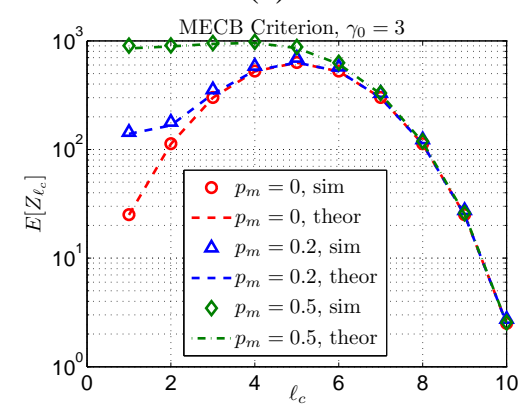

(f)

Figure 9: $\mathrm{E}\left[Z_{\ell_{s}}\right], \mathrm{E}\left[Z_{\ell_{p}}\right]$, and $\mathrm{E}\left[Z_{\ell_{c}}\right]$ as a function of (a) $\ell_{s}$, (b) $\ell_{p}$ and (c) $\ell_{c}$ when $n=m=10$ under a binomial PR activity model, and for various values of the probability of channel occupancy $p$. For figures (a), (b), and (c), perfect channel state information is assumed. For figures (d), (e) and (f), the probability of a channel being occupied by a PR is set to $p=0.3$. Imperfect channel state information is assumed. The probability of false alarm is fixed to $p_{f}=0.1$, while the probability of mis-detection is varied.

\subsection{Expected Number of Clusters of Size $Z_{\ell}$.}

We first evaluated the expected number of bicliques of size $\ell$, where $\ell$ is defined according to the MNB, MEB or MECB criteria. For each realization of a bipartite graph generated under a fixed set of parameters $\left(p, p_{m}, p_{f}\right)$, we counted the number of possible distinct bicliques of size $\ell$. We then averaged over 10,000 bipartite graph realizations with the same parameters and compared the outcome with the values obtained theoretically from propositions $1,2,3$, and 4 .

Figures $9(\mathrm{a}), 9(\mathrm{~b})$, and $9(\mathrm{c})$ show $\mathrm{E}\left[Z_{\ell_{s}}\right], \mathrm{E}\left[Z_{\ell_{p}}\right]$, and $\mathrm{E}\left[Z_{\ell_{c}}\right]$, as a function of $\ell_{s}, \ell_{p}$, and $\ell_{c}$, respectively, when perfect channel state information is assumed. The y-axis is shown in logarithmic scale due to the wide range of values obtained for $\mathrm{E}\left[Z_{\ell_{s}}\right], \mathrm{E}\left[Z_{\ell_{p}}\right]$, and $\mathrm{E}\left[Z_{\ell_{c}}\right]$. We observe that the values obtained from the simulations agree with the theoretical ones. Moreover, clusters of better quality (larger $\ell_{s}, \ell_{p}$ and $\ell_{c}$ ) are possible with the increase of the probability of a channel to be idle, expressed by $p$. Note in figure 9(b) that not all values of $\ell_{p}$ are possible, because $\ell_{p}$ is a product of two integers. In fact any $\ell_{p}$ which is an odd prime larger than $\max \{n, m\}$ is not feasible. In addition, $\ell_{p}$ 's with factors larger than $\max \{n, m\}$ are also not feasible. The number of factor decompositions of a particular $\ell_{p}$, affects the number of possible bicliques that can be constructed (more factor decompositions possible, means more $|X|,|Y|$ combinations that yield a particular $\ell_{p}$ ). This fact explains the "sawtooth" behavior of $\mathrm{E}\left[Z_{\ell_{p}}\right]$.

In figures $9(\mathrm{~d}), 9(\mathrm{e})$ and $9(\mathrm{f})$, we show $\mathrm{E}\left[Z_{\ell_{s}}\right], \mathrm{E}\left[Z_{\ell_{p}}\right]$, and $\mathrm{E}\left[Z_{\ell_{c}}\right]$, under the imperfect channel state information. In this set of experiments, we set the probability of a channel to be idle to $p=0.3$, the probability of false alarm to $p_{f}=0.1$ and varied the probability of mis-detection $p_{m}$. From figures 9(d) and 9(e), we observe that the MNB and MEB criteria are insensitive to events of mis-detection. This is 


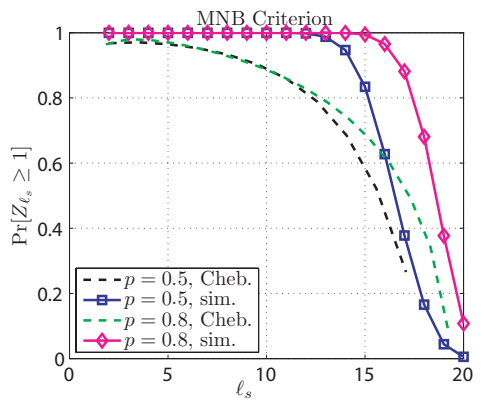

(a) $p_{f}=p_{m}=0$.

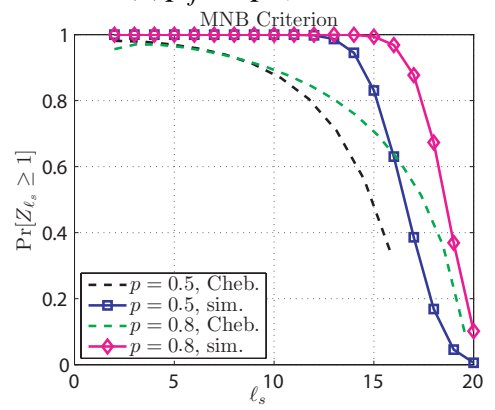

(d) $p_{f}=0.1, p_{m}=0.2$.

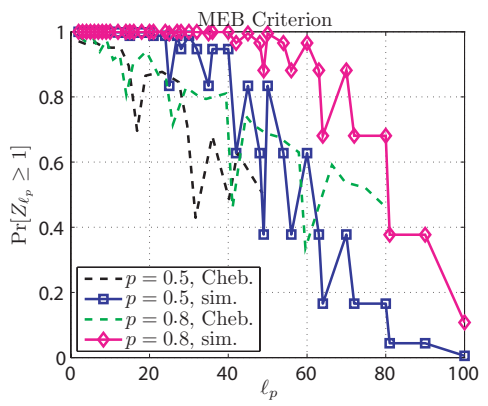

(b) $p_{f}=p_{m}=0$.

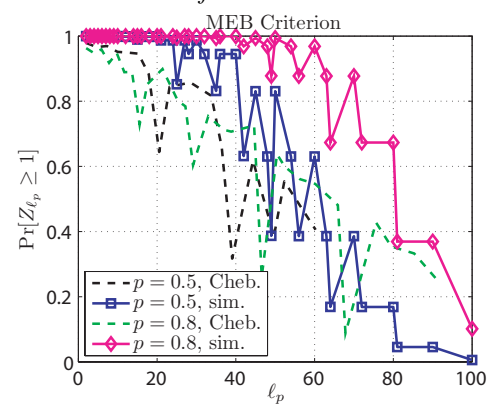

(e) $p_{f}=0.1, p_{m}=0.2$.

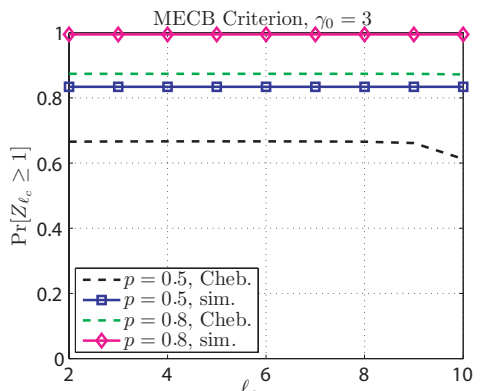

(c) $p_{f}=p_{m}=0$.

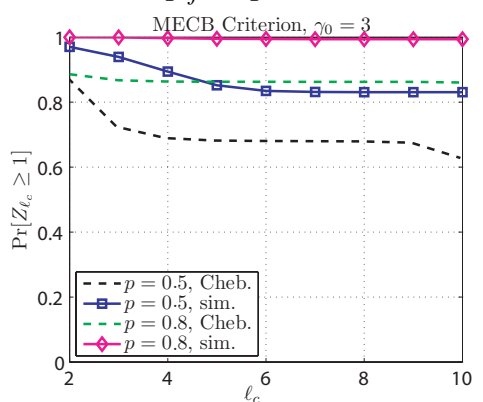

(f) $p_{f}=0.1, p_{m}=0.2$.

Figure 10: Probability of existence for a biclique of size $\ell_{s}, \ell_{p}$ and $\ell_{c}$ based on simulation, and based on the Chebyshev inequality.

because, these events occur independently on every channel and for every CR. Hence, for typical values of $p_{m}$ it is highly unlikely that the same channel is mis-detected on several CRs, an event that would impact the cluster construction. On the other hand, for the MECB criterion, high values of $p_{m}$, increase the number of possible clusters of small size, since it is possible that CRs forming such clusters, mis-detect the same set of channels. However, for clusters of larger size, mis-detection events have practically no impact.

From figures 9(a)-9(f), we can derive the cluster with biclique size $\ell^{*}$ for which $\mathrm{E}\left[Z_{\ell^{*}}\right]=1$. The value $\ell^{*}$ denotes the maximum biclique size which can be constructed under fixed parameters $\left(n, m, p, p_{f}, p_{m}\right)$, and, on average, appears once for every instance of PR activity. Note the an expected value of $E\left[Z_{\ell^{*}}\right]=1$, does not guarantee that a cluster with biclique size $\ell^{*}$ will be obtained with a high probability. This probability is evaluated in the following section.

\subsection{Probability of Existence of a Cluster of size $\ell$}

In this section, we evaluate the probability of existence of a cluster of a size $\ell$. We compare the values obtained via simulations to those computed by the Chebyshev inequality, as expressed in (10). The computation of the Chebyshev inequality is limited to values for which $\mathrm{E}\left[Z_{\ell}\right]>1$, and $\frac{\operatorname{Var}\left(\mathrm{E}\left[Z_{\ell}\right]\right)}{\left(\mathrm{E}\left[Z_{\ell}\right]-1\right)^{2}} \leq 1$. In figures 10 (a)-(c), we show the probability that at least one cluster of size $\ell$ exists, for values of $p=0.5$ and $p=0.8$, under perfect channel state information. This probability, denoted $\operatorname{as} \operatorname{Pr}\left[Z_{\ell} \geq 1\right]$, is plotted as a function of the size $\ell$, for each of the three clustering criteria. In particular, figure 10 (a) corresponds to the MNB criterion, figure 10(b) corresponds to the MEB criterion and figure 10(c) corresponds to the MECB criterion. For the MNB criterion, the Chebyshev bound provides a good approximation of $\operatorname{Pr}\left[Z_{\ell} \geq 1\right]$, for small values of $\ell_{s}$, but becomes loose with the increase of $\ell_{s}$. Similar behavior is observed for the case of the MEB criterion. Nonetheless, the theoretical bound of $\operatorname{Pr}\left[Z_{\ell} \geq 1\right]$, allows the estimation of the cluster sizes in terms of $\ell$ before the network deployment, when the PR activity model is known. For the case of 


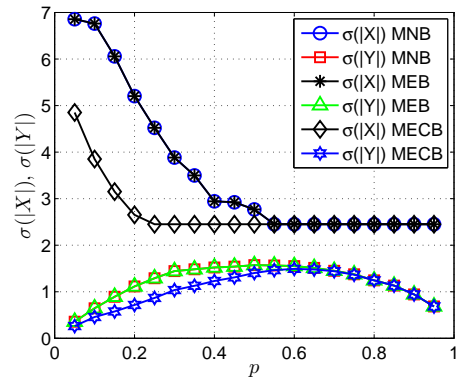

(a) $p_{f}=p_{m}=0$.

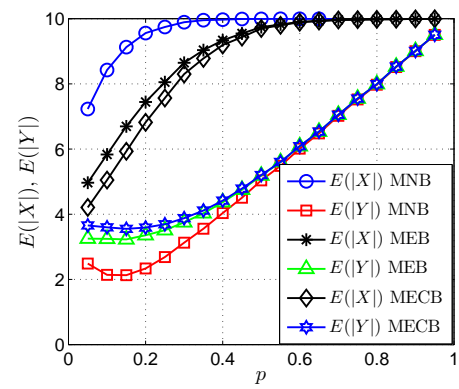

(d) $p_{f}=0.1, p_{m}=0.2$.

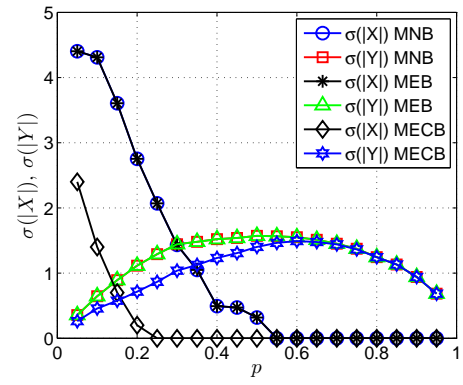

(b) $p_{f}=p_{m}=0$.

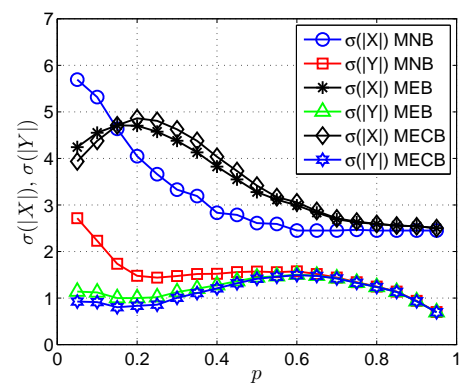

(e) $p_{f}=0.1, p_{m}=0.2$.

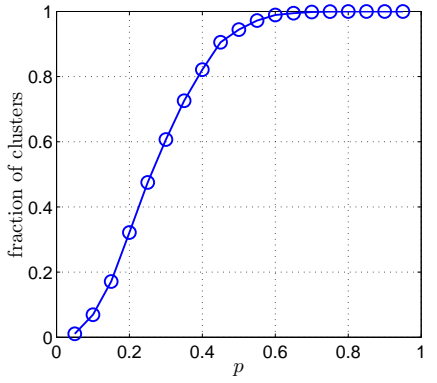

(c) $p_{f}=p_{m}=0$.

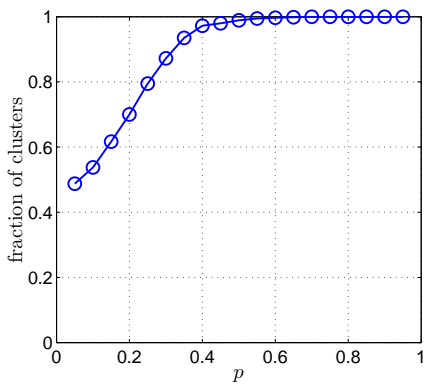

(f) $p_{f}=0.1, p_{m}=0.2$.

Figure 11: Comparison of the MNB, MEB and MECB clustering criteria under perfect and imperfect channel state information.

the MECB criterion, we observe that the Chebyshev bound stays within a $10 \%$ margin from the actual value of $\operatorname{Pr}\left[Z_{\ell_{c}} \geq 1\right]$, thus providing a more accurate prediction of the possible cluster sizes. Note that $\operatorname{Pr}\left[Z_{\ell_{c}} \geq 1\right]$ is almost constant for $p=0.8$ since at least $\gamma_{0}=3$ channels are idle to all neighboring CRs with almost certainty.

Figures 10(d)-(f) show $\operatorname{Pr}\left[Z_{\ell} \geq 1\right]$, as a function of $\ell$ for the three clustering criteria under imperfect channel state information. For this set of experiments, the false alarm probability and the mis-detection probability where set to $p_{f}=0.1$ and $p_{m}=0.2$, respectively. No significant change is observed for the MNB and the MEB criteria, which almost yield identical values as in figures 10(a) and 10(c). However, under the MECB criterion, we observe that probability of constructing small clusters (up to 4 nodes), with at least $\gamma_{0} \geq 5$ channels in common increases when $p=0.5$, compared to the values shown in 10(c). This is because the events of mis-detection contribute to the perceived set of idle channels, causing several neighboring CRs to surpass the threshold $\gamma_{0}$ for forming a cluster. The effect of channel state mis-detection tapers off for larger values of $\ell_{c}$, since mis-detection events occur independently on each CR and on each channel.

\subsection{Clustering Performance}

In this section, we evaluate the performance of the clustering algorithm presented in Section 5. We deployed a network of $100 \mathrm{CRs}$ within a $1 \mathrm{Km} \times 1 \mathrm{Km}$ area, which coexisted with a set of $10 \mathrm{PRs}$. A total of 10 channels were licensed to the PRs. The PR activity followed the binomial model in (2). PRs were assigned a communication range of $250 \mathrm{~m}$, while the communication range of the CRs varied to allow for different values on the probabilities $p_{c}, p_{w}$. We applied the DCA-based clustering algorithm and compared the MNB, MEB and MECB criteria in terms of the expected cluster size and the expected number of common idle channels per cluster. Our simulations were repeated for 100 network realizations to ensure statistical 
validity.

In figure 11(a), we compare the average cluster size $\mathrm{E}(|X|)$ and the average number of idle common channels $\mathrm{E}(|Y|)$ for the MNB, MEB and MECB criteria, under perfect channel state information, and as a function of the PR activity parameter $p$. The communication range of CRs was set to $150 \mathrm{~m}$. We observe that the MNB and MEB criteria yield identical performance. This is because when the spectrum opportunities are perfectly known and the range of the CRs is almost identical to that of PRs, the bipartite graphs constructed by CRs are almost bicliques. Thus both the MNB and MEB criteria yield the same clusters.

We also observe a graceful trade off between $\mathrm{E}(|X|)$ and $\mathrm{E}(|Y|)$, as a function of the PR activity (expressed by $p$ ). When PR activity is high and idle channels are sparse, the cluster size $|X|$ is reduced in order to maintain an acceptable number of common idle channels within each cluster. On the other hand, when PR activity is low, a larger cluster size is favored. This trade off is also captured in the standard deviation of $\mathrm{E}(|X|)$ and $\mathrm{E}(|Y|)$ as a function of $p$, which is shown in figure 11(b). When the PR activity is high, the spatial variation of the idle channel becomes large. In turn, this leads to a large value for $\sigma(|X|)$ to accommodate for the lack of idle channels at different localities. On the other hand, when the PR activity is low (most channels are idle), clusters are primarily decided based on the physical topology. Hence, for high values of $p, \mathrm{E}(|X|)$ and $\sigma(|X|)$ converge to their average values in the absence of any PR activity.

On the other hand, the trade off between $\mathrm{E}(|X|)$ and $\mathrm{E}(|Y|)$ is managed differently by the MECB criterion. The latter favors clusters of maximum size as long as the threshold $\gamma_{0}$ is satisfied ( $\gamma_{0}$ was set to 3 in our experiments). From figure 11(a), we observe that the MECB criterion yields higher values of $E(|X|)$ compared to the MNB and MEB criteria, for the same value of $p$. Moreover, $\mathrm{E}(|Y|)$ is kept above the threshold $\gamma_{0}$. Finally, the MECB criterion yields smaller values for both $\sigma(|X|)$ and $\sigma(|Y|)$, because it does not try to balance between $|X|$ and $|Y|$, but maximize $|X|$ while $|Y| \geq \gamma_{0}$.

However, the MECB criterion is not always successful in constructing a valid cluster, due to low idle channel availability (failed attempts are excluded from the graphs in 11(a), and 11(b)). In figure 11(c), we show the fraction of clusters that the MECB criterion is able to construct, when individual CRs attempt to perform clustering based on their bipartite graphs. We note that for small values of $p$, only a fraction of nodes are able to form clusters with $|Y| \geq \gamma_{0}$. For cases where a cluster satisfying the threshold $\gamma_{0}$ cannot be formed, the value of $\gamma_{0}$ must be lowered to produce a valid clustering.

We also conducted the same set of experiments by considering a smaller CR communication range $(r=50)$, a false alarm probability of $p_{f}=0.1$ and a mis-detection probability of $p_{m}=0.2$. All this factors increase the dissimilarity between the channel lists of neighboring CRs, thus testing the adaptability of our clustering algorithm to the spacial variation of PR activity. In figures 11(d), 11(e), and 11(f), we show $\mathrm{E}(|X|), \mathrm{E}(|Y|), \sigma(|X|), \sigma(|Y|)$, and the fraction of clusters successfully constructed by the MECB criterion as a function of the PR activity $p$. From figures 11(d), 11(e), we can reason that the MNB criterion favors the creation of clusters of larger size at the expense of a smaller number of common idle channels. The standard deviation $\sigma(|X|)$ becomes very small as $p$ increases, while $\sigma(|Y|)$ maintains a high value. The MEB criterion results in a better balance between $|X|$ and $|Y|$. For the same value of $p$, the MEB criterion constructs clusters of smaller size compared to the MNB criterion, but with larger number of common idle channels. In addition, both standard deviations $\sigma(|X|)$ and $\sigma(|Y|)$ maintain a small value for every $p$, illustrating the stability of the clusters constructed. The MECB criterion performs similar to the MEB criterion. From this set of experiments, we can conclude that the MEB and MECB criteria are less sensitive to the heterogeneity of the idle channel lists of neighboring CRs, leading to a favorable management of the trade off between $|X|$ and $|Y|$. 


\section{Related Work}

Clustering algorithms for CRNs have been proposed in the context of facilitating basic network operations such as control channel establishment [11, 12, 41, 42], MAC protocol implementation [11, 41], spectrum management [43, 44], and cooperative spectrum sensing [40]. Clustering is an essential architectural element for reducing the CRN management operation to managing a collection of smaller CR groups.

Zhao et. al. proposed a distributed coordination protocol for opportunistically sharing the dynamic spectrum [11]. In this protocol, CRs self-organize into groups (clusters) based on the similarity between their lists of idle channels. CRs are grouped in the same cluster as long as they share at least one common idle channel, and a multi-hop route exists between all CRs of the same cluster. CRs at the boundary of clusters may belong to multiple clusters, and serve as gateways for inter-cluster coordination. The main objective of the method in [11] is to minimize the number of clusters in the entire network, in order to reduce the overhead associated with topology management and CR coordination. To address the dynamic nature of spectrum availability, CRs "swiftly" migrate to an idle channel, if the currently used one is occupied by a PR. Given that the minimum number of common idle channels allowed per cluster is one, clusters sharing only one channel are possible. For such clusters, reclustering can become a frequent operation in the light of high PR activity.

Chen et. al. proposed the CogMesh architecture for addressing the problem of control channel assignment in CRNs [41]. In CogMesh, the CRN is partitioned into clusters based on the local channel availability. The distributed cluster formation algorithm is based on the initial assignment of few nodes as clusterheads $(\mathrm{CHs})$, responsible for creating clusters. The rest of the nodes join adjacent $\mathrm{CHs}$ with which they share the largest number of common idle channels. To adapt to PR activity, clusters are split to smaller ones if no common idle channel is available at any given point. At a later stage clusters may merge if channel availability permits it. Baddour et. al. proposed a clustering algorithm for CRNs based on affinity propagation [42]. As in the case of [41], several CRs are declared to be the CHs, with nearby nodes joining clusters based on the similarity of their idle channel lists with those of the $\mathrm{CH}$. The convergence of the algorithm to the same cluster memberships was proved to occur in a decentralized manner.

Asterjadhi et al. proposed the Combo algorithm for partitioning a CRNs into mutually disjoint clusters based on the local spectrum availability [45]. The distinct feature of this clustering algorithm is the fact that clusters can span up to k-hops (as opposed to one-hop clusters created by the mechanisms in [11, 12, 41]). The mechanism in [45] relies on the election of a $\mathrm{CH}$ node based on a weighted priority key which consolidates connectivity with local spectrum opportunities. Simulation results showed that the Combo algorithm maintains a large number of common idle channels within each cluster while keeping the number of CRs composing each cluster large.

In [12], we proposed a distributed clustering algorithm named SOC, which addressed the problem of dynamic control channel assignment in CRNs. The goal of the clustering was to ensure a sufficient number of common idle channels for the establishment and maintenance of a broadcast control channel within each cluster. In SOC, clusters are formed based on the joint representation of the network topology and idle spectrum as a bipartite graph. The same mapping is used as the basis for our present work. We showed that clusters can be mapped to biclique graphs (complete subgraphs of bipartite graphs) in which one side of the bipartition represents the cluster membership, while the other side represents the set of common idle channels within the cluster. In addition, we showed that by exchanging individual sensing observations of the idle spectrum, CRs were guaranteed to converge to the same cluster memberships in a distributed manner. Compared to [12], our goal is to analytically evaluate the performance of different clustering criteria which rely on the biclique construction. Given a PR activity model and a spectrum sensing model, we investigate the problem of determining the existence of clusters (bicliques) of different sizes and the 
expectation on the cluster size and number of common idle channels per cluster.

The problem of clustering has been extensively studied in the context of wireless ad hoc networks under a fixed spectrum paradigm (e.g., [35, 36, 46-49]). Clustering methods for ad hoc networks can be classified to $\mathrm{CH}$-first and cluster-first. In $\mathrm{CH}$-first methods, a set of nodes are initially selected as $\mathrm{CHs}$, based on a metric (or combination of metrics) of interest such as connectivity degree, remaining energy, id, etc. The rest of the nodes attach to a $\mathrm{CHs}$ to form the final clusters. In cluster-first methods, the clusters are collaboratively formed before a $\mathrm{CH}$ can be elected within each cluster. Interested reader is referred to [46-48] and the references therein for clustering methods proposed for wireless ad hoc networks.

\section{Conclusions}

We addressed the problem of managing the spatial variation of spectrum opportunities in CRNs, by proposing spectrum-aware clustering criteria. These criteria were based on the joint representation of the physical topology and spectrum availability as bipartite graphs. We mapped the problem of constructing spectrum-aware clusters to the problem of constructing bicliques of maximum size from the bipartite graphs. Three clustering criteria were proposed based on three instances of the biclique construction problem. We further showed how our clustering criteria can be combined with distributed coordination mechanisms for forming clusters in CRNs. For each criterion, we analytically evaluated the expected clustering performance under various models of PR activity and channel state information. Our evaluations show that the proposed criteria, lead to a balance between the size of the constructed clusters and the number of common idle channels within each cluster. This is a desirable feature for ensuring moderate overhead for the topology management of the CRN and the availability of sufficient bandwidth for intra-cluster communication.

\section{Acknowledgments}

Milan Bradonjić was supported in part by the Department of Energy ASCR program. Loukas Lazos was supported in part by the National Science Foundation (under NSF grants CAREER CNS-0844111, CNS-1016943). Any opinions, findings, conclusions, or recommendations expressed in this paper are those of the author(s) and do not necessarily reflect the views of NSF.

\section{References}

[1] T. Rappaport, Wireless communications: principles and practice, Prentice Hall PTR Upper Saddle River, NJ, USA, 2001.

[2] D. Tse, P. Viswanath, Fundamentals of Wireless Communication, Cambridge University Press, 2005.

[3] M. McHenry, D. McCloskey, New York City spectrum occupancy measurements (September 2004).

[4] M. A. McHenry, P. A. Tenhula, D. McCloskey, D. A. Roberson, C. S. Hood, Chicago spectrum occupancy measurements \& analysis and a long-term studies proposal, in: Proceedings of the first international workshop on Technology and policy for accessing spectrum, 2006.

[5] FCC, Second memorandum opinion and order, FCC-10-174 (2010).

[6] I. Akyildiz, W. Lee, M. Vuran, S. Mohanty, Next generation/dynamic spectrum access/cognitive radio wireless networks: A survey, Computer Networks 50 (13) (2006) 2127-2159.

[7] S. Mishra, A. Sahai, R. Brodersen, Cooperative sensing among cognitive radios, in: Proceedings of the International Conference on Communications, ICC, 2006.

[8] A. Ghasemi, E. Sousa, Collaborative spectrum sensing for opportunistic access in fading environments, in: Proceedings of the IEEE International Symposium on New Frontiers in Dynamic Spectrum Access Networks, (DYSPAN), 2005, pp. $131-136$.

[9] G. Ganesan, Y. Li, Cooperative spectrum sensing in cognitive radio networks, in: Proceedings of the IEEE International Symposium on New Frontiers in Dynamic Spectrum Access Networks, (DYSPAN), 2005, pp. 137-143.

[10] P. Bahl, R. Chandra, T. Moscibroda, R. Murty, M. Welsh, White space networking with Wi-Fi like connectivity, ACM SIGCOMM Computer Communication Review 39 (4) (2009) 27-38. 
[11] J. Zhao, H. Zheng, G.-H. Yang, Spectrum sharing through distributed coordination in dynamic spectrum access networks, Wireless Communications and Mobile Computing 7 (9) (2007) 1061-1075.

[12] L. Lazos, S. Liu, M. Krunz, Spectrum opportunity-based control channel assignment in cognitive radio networks, in: Proceedings of the 6th Annual IEEE Communications Society Conference on Sensor, Mesh and Ad Hoc Communications and Networks, 2009, pp. 1-9.

[13] S. Geirhofer, L. Tong, B. Sadler, A measurement-based model for dynamic spectrum access in WLAN channels, in: Proceedings of IEEE MILCOM, 2006.

[14] A. Motamedi, A. Bahai, MAC protocol design for spectrum-agile wireless networks: Stochastic control approach, in: Proceedings of the 2nd IEEE International Symposium on New Frontiers in Dynamic Spectrum Access Networks, (DYSPAN), 2007, pp. 448-451.

[15] Q. Zhao, L. Tong, A. Swami, Y. Chen, Decentralized cognitive MAC for opportunistic spectrum access in Ad Hoc networks: A POMDP framework, IEEE Journal on Selected Areas in Communications 25 (3) (2007) 589-600.

[16] H. Kim, K. Shin, Efficient discovery of spectrum opportunities with MAC-layer sensing in cognitive radio networks, IEEE Transactions on Mobile Computing (2007) 533-545.

[17] L. Yang, L. Cao, H. Zheng, Proactive channel access in dynamic spectrum networks, Physical Communication 1 (2) (2008) $103-111$.

[18] S. Tang, W. Li, Performance analysis of a channel allocation scheme for multi-service mobile cellular networks, International Journal of Communication Systems 20 (2) (2007) 177-205.

[19] W. Li, X. Chao, Modeling and performance evaluation of a cellular mobile network, IEEE/ACM Transactions on Networking (TON) 12 (1) (2004) 145-157.

[20] Y. Fang, I. Chlamtac, Y. Lin, Channel occupancy times and handoff rate for mobile computing and pcs networks, IEEE Transactions on Computers 47 (6) (1998) 679.

[21] L. Kleinrock, Queueing Systems: Volume 2: Computer Applications, John Wiley \& Sons New York, 1976.

[22] E. Pinsky, A simple approximation for the Erlang loss function, Performance Evaluation 15 (3) (1992) 155-161.

[23] L. Xiao, L. Greenstein, N. Mandayam, W. Trappe, Fingerprints in the ether: Using the physical layer for wireless authentication, in: Proceedings of the IEEE International Conference on Communications (ICC), 2007, pp. 4646-4651.

[24] S. Tang, B. Mark, Modeling and analysis of opportunistic spectrum sharing with unreliable spectrum sensing, IEEE Transactions on Wireless Communications 8 (4) (2009) 1934-1943.

[25] Y. Gao, Y. Jiang, Performance analysis of a cognitive radio network with imperfect spectrum sensing, in: Proceedings of INFOCOM Mini Conference, 2010, pp. 1-6.

[26] S. Ciftci, M. Torlak, A comparison of energy detectability models for spectrum sensing, in: Proceedings of the IEEE Global Telecommunications Conference (GLOBECOM), 2008, pp. 1-5.

[27] C. Bettstetter, On the connectivity of Ad Hoc networks, The Computer Journal 47 (4) (2004) 432.

[28] X. Huang, N. Han, G. Zheng, S. Sohn, J. Kim, Weighted-collaborative spectrum sensing in cognitive radio, in: Proceedings of CHINACOM, 2007, pp. 110-114.

[29] M. Dawande, P. Keskinocak, S. Tayur, On the biclique problem in bipartite graphs, Tech. rep., GSIA Working Paper 1996-04, Carnegie Mellon University (1996).

[30] M. Dawande, P. Keskinocak, J. Swaminathan, S. Tayur, On bipartite and multipartite clique problems, Journal of Algorithms 41 (2) (2001) 388-403.

[31] M. Yannakakis, Node-deletion problems on bipartite graphs, SIAM Journal on Computing 10 (1981) 310-320.

[32] D. Hochbaum, Approximating clique and biclique problems, Journal of Algorithms 29 (1) (1998) 174-200.

[33] R. Peeters, The maximum edge biclique problem is NP-complete, Discrete Applied Mathematics 131 (3) (2003) $651-654$.

[34] S. Madeira, A. Oliveira, Biclustering algorithms for biological data analysis: a survey, IEEE Transactions on computational Biology and Bioinformatics (2004) 24-45.

[35] S. Basagni, Distributed clustering for Ad Hoc networks, in: Proceedings of the IEEE International Symposium on Parallel Architectures, Algorithms, and Networks I-SPAN, 1999, pp. 310-315.

[36] M. Chatterjee, S. Das, D. Turgut, WCA: A weighted clustering algorithm for mobile Ad Hoc networks, Cluster Computing 5 (2) (2002) 193-204.

[37] C. Lin, M. Gerla, Adaptive clustering for mobile wireless networks, Selected Areas in Communications, IEEE 15 (7) (1997) $1265-1275$.

[38] J. Yu, P. Chong, A survey of clustering schemes for mobile Ad Hoc networks, IEEE Communications Surveys and Tutorials 7 (1) (2005) 32-48.

[39] S. Basagni, Distributed and mobility-adaptive clustering for ad hoc networks, Tech. rep., Erik Johnson School of Engineering and Computer Science, The University of Texas at Dallas (July 1998).

[40] C. Sun, W. Zhang, K. Letaief, Cluster-based cooperative spectrum sensing in cognitive radio systems, in: Proceedings of the IEEE International Conference on Communications, (ICC), 2007, pp. 2511-2515. 
[41] T. Chen, H. Zhang, G. Maggio, I. Chlamtac, CogMesh: a cluster-based cognitive radio network, in: Proceedings of the IEEE International Symposium on New Frontiers in Dynamic Spectrum Access Networks, (DYSPAN), 2007, pp. 168-178.

[42] K. E. Baddour, O. Ureten, T. J. Willink, Efficient clustering of cognitive radio networks using affinity propagation, in: Proceedings of 18th International Conference on Computer Communications and Networks (ICCCN), 2009, pp. 1-6.

[43] A. Alsarhan, A. Agarwal, Cluster-based spectrum management using cognitive radios in wireless mesh networks, in: Proceedings of 18th International Conference on Computer Communications and Networks (ICCCN), 2009, pp. 1-6.

[44] P. Pawelczak, C. Guo, R. Prasad, R. Hekmat, Cluster based spectrum sensing architecture for opportunistic spectrum access networks, Tech. rep., Delft University of Technology (Feb 2006).

[45] A. Asterjadhi, N. Baldo, M. Zorzi, A cluster formation protocol for cognitive radio Ad Hoc networks, in: European Wireless Conference (EW), 2010, pp. 955-961.

[46] J. Y. Yu, P. H. J. Chong, A survey of clustering schemes for mobile Ad Hoc networks, IEEE Communications Surveys \& Tutorials 7 (1) (2005) 32-48.

[47] Y. P. Chen, A. L. Liestman, L. J., Clustering algorithms for Ad Hoc wireless networks, Ad Hoc and Sensor Networks.

[48] A. A. Abbasi, M. Younis, A survey on clustering algorithms for wireless sensor networks, Computer Communications 30 (1415) (2007) $2826-2841$.

[49] O. Younis, S. Fahmy, HEED: a hybrid, energy-efficient, distributed clustering approach for Ad Hoc sensor networks, IEEE Transactions on Mobile Computing (2004) 366-379.

[50] M. Bradonjić, A. Hagberg, A. Percus, The structure of geographical threshold graphs, Internet Mathematics 5 (1-2) (2008) $113-140$.

\section{Appendix A. Computation of $p_{c}$ in (5)}

The value of $p_{c}$ expresses the probability of a CR $z$ sensing a channel $i$ occupied by a PR $x$ as idle, given that a neighboring CR $y$, also senses $i$ as idle. This probability is a measure of the correlation between the sensing observations of neighboring nodes. Probability $p_{c}$ is given by:

$$
\begin{aligned}
\operatorname{Pr}\left[d_{x z}>R \mid d_{x y}>R, d_{y z} \leq r\right] & =1-\operatorname{Pr}\left[d_{x z} \leq R \mid d_{x y}>R, d_{y z} \leq r\right] \\
& =1-\frac{\operatorname{Pr}\left[d_{x z} \leq R, d_{x y}>R \mid d_{y z} \leq r\right]}{\operatorname{Pr}\left[d_{x y}>R\right]}
\end{aligned}
$$

The numerator of (A.2) is computed based on Figure A.12. Here, we are interested in the event that CR $y$ is outside the range $R$ of $\mathrm{PR} x$ while $\mathrm{CR} z$ is within the range of $x$, under the constraint that $y, z$ are one-hop neighbors. The probability of such an event depends on the size of the intersection area between a disk of radius $R$ and a disk of radius $r$, when the centers of these disks are located at a distance $d_{x y}$ apart. This area, denoted by $A_{\text {in }}$ is given by $[27,50]$

$$
A_{\text {in }}=r^{2} \cos ^{-1}\left(\frac{R^{2}-r^{2}-d_{x y}^{2}}{2 r d_{x y}}\right)+R^{2} \cos ^{-1}\left(\frac{R^{2}-r^{2}+d_{x y}^{2}}{2 R d_{x y}}\right)-\frac{1}{2} r R \sqrt{4-\frac{\left(d_{x y}^{2}-R^{2}-r^{2}\right)^{2}}{r^{2} R^{2}}} .
$$

The numerator of (A.2) becomes

$$
\begin{aligned}
\operatorname{Pr}\left[d_{x z} \leq R, d_{x y}>R \mid d_{y z} \leq r\right] & =\int_{\xi=R}^{R+r} \operatorname{Pr}\left[z \in A_{i n}(\xi), \mid d_{x y}=\xi\right] \operatorname{Pr}\left[d_{x y}=\xi\right] d \xi \\
& =\int_{\xi=R}^{R+r} \frac{A_{i n}(\xi)}{\pi r^{2}} \frac{2 \xi}{R^{2}} d \xi .
\end{aligned}
$$

In (A.5), we consider all values of $d_{x y}=\xi$ for which $R<d_{x y}<R+r$ so that the disks of radius $R$ 


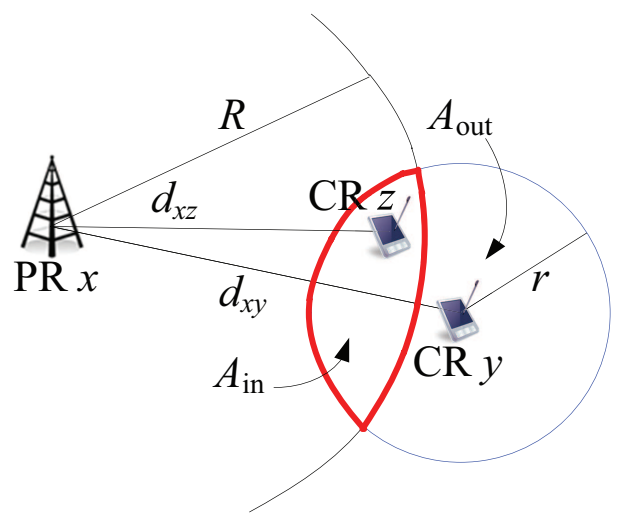

Figure A.12: CR $y$ and $\mathrm{CR} z$ are one-hop neighbors. The event of $\mathrm{CR} z$ being within the range of PR $x$, while $\mathrm{CR} y$ being outside the range of $x$.

and $r$ intersect, and then compute the probability that $z$ which is constrained to be within a radius $r$ from $y$ is located within the area $A_{i n}$. In this scenario, a channel $i$ occupied by PR $x$ is sensed idle by CR $y$ but occupied by $z$. Substituting (A.5) to (A.2) yields the desired probability value.

\section{Appendix B. Proof of Proposition 1}

Proof. From the definition of $\mathrm{E}\left[Z_{\ell_{s}}\right]$ it follows that,

$$
\begin{aligned}
\mathrm{E}\left[Z_{\ell_{s}}\right]= & \sum_{X \subseteq \mathcal{A}, Y \subseteq \mathcal{B},|X|+|Y|=\ell_{s},|X|,|Y| \geq 1} \operatorname{Pr}\left[I_{(X, Y)}\right] \\
= & \sum_{i \geq 0} \sum_{X \subseteq \mathcal{A}, Y \subseteq \mathcal{B},|X|+|Y|=\ell_{s},|X|,|Y| \geq 1} \operatorname{Pr}\left[I_{(X, Y)} \mid N=i\right] \operatorname{Pr}[\mathrm{N}=i] \\
= & \sum_{i=0}^{\min \left\{m, \ell_{s}-1\right\}} \operatorname{Pr}[\mathrm{N}=i] \sum_{\alpha=0}^{\min \left\{i, \ell_{s}-1\right\}} \sum_{\beta=\max \left\{0, \ell_{s}-n-\alpha\right\}}^{\min \left\{\ell_{s}-1-\alpha, m-i\right\}} C\left(n, \ell_{s}-\alpha-\beta\right) C(i, \alpha) C(m-i, \beta) \\
& \times \operatorname{Pr}\left[\left(\ell_{s}-\alpha-\beta\right) \beta \text { false est. }\right] .
\end{aligned}
$$

In (B.1b), we condition upon the probability that the number of idle channels $N$ is equal to a fixed value $i$, and sum over all possible values of $i$. Let $\mathcal{D} \subseteq \mathcal{B}$ denote the set of vertices corresponding to the $i$ idle channels. The vertices in $\mathcal{D}$ are connected to all vertices in $\mathcal{A}$ (idle channels are perfectly sensed). In addition, vertices from the remaining set $\mathcal{B} \backslash \mathcal{D}$ are independently connected to vertices in $X$, due to false channel estimation. When $\alpha$ vertices from $\mathcal{D}$ and $\beta$ vertices from $\mathcal{B} \backslash \mathcal{D}$ connect to the same $\left(\ell_{s}-\alpha-\beta\right)$ vertices of $\mathcal{A}$, we obtain a biclique of size $\ell_{s}$. The $\left(\ell_{s}-\alpha-\beta\right) \beta$ edges which appear on the bipartite graph due to false channel estimation exist with probability $\operatorname{Pr}\left[\left(\ell_{s}-\alpha-\beta\right) \beta\right.$ false est. $]=\Pi_{S}^{t}(\alpha, \beta)$. Here, the probability $\Pi_{S}^{t}(\alpha, \beta)$ of the appearance of a clique of size $\ell_{s}$ depends on the PR activity and channel state information models:

$$
\Pi_{S}^{t}\left(\ell_{s}, \alpha, \beta\right)=\left\{\begin{array}{l}
1, \quad(\mathrm{i}), \\
p_{w}^{\beta} p_{c}^{\beta\left(\ell_{s}-\alpha-\beta-1\right)},(\mathrm{ii}), \\
\left(1-p_{f}\right)^{\left(\ell_{s}-\alpha-\beta\right) \alpha} p_{m}^{\left(\ell_{s}-\alpha-\beta\right) \beta}, \quad(\mathrm{iii}), \\
\left.\left(1-p_{f}\right)^{\left(\ell_{s}-\alpha-\beta\right) \alpha}\left(\left(1-p_{w}\right) p_{m}+p_{w}\left(1-p_{f}\right)\right)^{\beta}\left(\left(1-p_{c}\right) p_{m}+p_{c}\left(1-p_{f}\right)\right]\right)^{\beta\left(\ell_{s}-\alpha-\beta-1\right)}, \text { (iv), }
\end{array}\right.
$$


for (i) perfect channel state information, type-I topologies (ii) perfect channel state information, type-II topologies, (iii) imperfect channel state information, type-I topologies, and (iv) imperfect channel state information, type-II topologies, respectively. Summing over all possible combinations of $i, \alpha, \beta$, we obtain the expected number of bicliques of size $\ell_{s}$, which completes the proof.

Note that in the case (i) of proposition 1 , when $\Pi_{S}^{t}\left(\ell_{s}, \alpha, \beta\right)=1$, by using Vandermode's identity the expected number of bicliques of size $\ell_{s}$ simplifies:

$$
\mathrm{E}\left[Z_{\ell_{s}}\right]=\sum_{i=\max \left\{1, \ell_{s}-n\right\}}^{\min \left\{m, \ell_{s}-1\right\}} \operatorname{Pr}[\mathrm{N}=i]\left(C\left(n+i, \ell_{s}\right)-C\left(n, \ell_{s}\right)-C\left(i, \ell_{s}\right)\right) .
$$

\section{Appendix C. Proof of Proposition 2}

Proof.

$$
\begin{aligned}
\mathrm{E}\left[Z_{\ell_{p}}\right] & \triangleq \sum_{X \subseteq \mathcal{A}, Y \subseteq \mathcal{B},|X| Y Y \mid=\ell_{p}} \operatorname{Pr}\left[\mathrm{I}_{(X, Y)}\right] \\
& \left.=\sum_{i \geq 0} \sum_{X \subseteq \mathcal{A}, Y \subseteq \mathcal{B},|X| Y Y \mid=\ell_{p}} \operatorname{Pr}\left[\mathrm{I}_{(X, Y)} \mid N=i\right]=i\right] \operatorname{Pr}[\mathrm{N}=i] \\
& =\sum_{i=\max \left\{1,\left[\frac{\ell_{p}}{n}\right]\right\}}^{\min \left\{m, \ell_{p}\right\}} \operatorname{Pr}[\mathrm{N}=i] \sum_{j=\max \left\{1,\left[\frac{\ell_{p}}{n}\right]\right\}, \frac{\ell_{p}}{j} \in \mathbb{Z}^{+}}^{\min \left\{i, \ell_{p}\right\}} C\left(n, \frac{\ell_{p}}{j}\right) C(i, j) .
\end{aligned}
$$

In (C.1b), we condition upon the probability that the number of idle channels $N$ is equal to a fixed value $i$, and sum over all possible values of $i$. The vertices of $\mathcal{B}$ corresponding to the $i$ idle channels are connected to all vertices of $\mathcal{A}$ due to the perfect sensing assumption. In (C.1c), we enumerate all possible bicliques with $|Y|=j$ and $|X|=\frac{\ell_{p}}{j}$, under the constraints $1 \leq j \leq i$ and $1 \leq \frac{\ell_{p}}{j} \leq n$. These constraints can be combined to $\max \left\{1,\left\lceil\frac{\ell_{p}}{n}\right\rceil\right\} \leq j \leq \min \left\{i, \ell_{p}\right\}$. Note that not all values of $j$ are possible since $\frac{\ell_{p}}{j}$ must be a positive integer. Summing over all possible values of $i$ weighted over the probability $\operatorname{Pr}[\mathrm{N}=i]$ yields (C.1c). Here, the number of idle channels $i$ is limited by the maximum number of channels $m$. Moreover, $i$ can be at most $\ell_{p}$ if we are to construct a biclique of size $\ell_{p}$. Finally, at least $\left\lceil\frac{\ell_{p}}{n}\right\rceil$ vertices from $\mathcal{B}$ must be connected to all vertices in $\mathcal{A}$, for a biclique of size $\ell_{p}$ to be feasible. Combining all constraints for $i$ yields the summation limits shown in (C.1c), which completes the proof. 